LOUIS-SÉBASTIEN GUIMOND

ZUZANA MASÁKOVÁ

EDITA PELANTOVÁ

\title{
Combinatorial properties of infinite words associated with cut-and-project sequences
}

Journal de Théorie des Nombres de Bordeaux, tome 15, no 3 (2003), p. $697-725$

<http://www.numdam.org/item?id=JTNB_2003_15_3_697_0>

(C) Université Bordeaux 1, 2003, tous droits réservés.

L'accès aux archives de la revue "Journal de Théorie des Nombres de Bordeaux » (http://jtnb.cedram.org/) implique l'accord avec les conditions générales d'utilisation (http://www.numdam.org/conditions). Toute utilisation commerciale ou impression systématique est constitutive d'une infraction pénale. Toute copie ou impression de ce fichier doit contenir la présente mention de copyright.

\section{Numdam}

Article numérisé dans le cadre du programme

Numérisation de documents anciens mathématiques

http://www.numdam.org/ 


\title{
Combinatorial properties of infinite words associated with cut-and-project sequences
}

\author{
par LoUIS-SÉBASTIEN GUIMOND, ZUZANA MASÁKOVÁ et EDITA \\ PELANTOVÁ
}

\begin{abstract}
RÉSUMÉ. Le but de cet article est d'étudier certaines propriétés combinatoires des suites binaires et ternaires obtenues par "coupe et projection". Nous considérons ici le processus de coupe et projection en dimension deux où les sous-espaces de projection sont en position générale. Nous prouvons que les distances entre deux termes adjacents dans une suite ainsi obtenue prennent toujours soit deux soit trois valeurs. Une suite obtenue par coupe et projection détermine ainsi de manière naturelle une suite symbolique (mot infini) sur deux ou trois lettres. En fait ces suites peuvent aussi être obtenues comme codages d'échanges de deux ou trois intervalles. Du point de vue de la complexité, la construction par coupe et projection donne des mots de complexité $n+1, n+$ constante et $2 n+1$. Les mots binaires ont une complexité égale à $n+1$ et sont donc sturmiens. Les mots ternaires ont une complexité égale à $n+$ constante ou $2 n+1$. Une coupe et projection a trois paramètres dont deux spécifient les sous-espaces de projection, le troisième déterminant la bande de coupe. Nous classifions les triplets qui correspondent à des mots infinis combinatoirement équivalents.
\end{abstract}

ABSTRACT. The aim of this article is to study certain combinatorial properties of infinite binary and ternary words associated to cut-and-project sequences. We consider here the cut-and-project scheme in two dimensions with general orientation of the projecting subspaces. We prove that a cut-and-project sequence arising in such a setting has always either two or three types of distances between adjacent points. A cut-and-project sequence thus determines in a natural way a symbolic sequence (infinite word) in two or three letters. In fact, these sequences can be constructed also by a coding of a 2- or 3-interval exchange transformation. According to the complexity the cut-and-project construction includes words with complexity $n+1, n+$ const. and $2 n+1$. The words on two letter alphabet have complexity $n+1$ and thus are Sturmian. The

Manuscrit reçu le 22 octobre 2001. 
ternary words associated to the cut-and-project sequences have complexity $n+$ const. or $2 n+1$. A cut-and-project scheme has three parameters, two of them specifying the projection subspaces, the third one determining the cutting strip. We classify the triples that correspond to combinatorially equivalent infinite words.

\section{Introduction}

In this paper we study certain ternary generalizations of binary Sturmian sequences $[14,15]$. Sturmian sequences have several equivalent characterizations. One of them is using the so-called cut-and-project scheme. Consider a lattice $\mathbb{Z}^{2}$, a straight line $p: y=\alpha x$ with an irrational slope $\alpha$, and a strip parallel to the line $p$ of such a width that the intersection of the strip with the $x$-axis is a line-segment of length 1 . The orthogonal projection of lattice points in the strip on the line $p$ determines a point set in $\mathbb{R}$ with only two possible distances between neighbours. If we represent these distances by letters $A$ and $B$ respectively and arrange these letters according to the order of distances in the point set, we obtain a Sturmian infinite word.

Replacing the orthogonal projection in the above scheme by projection in any direction may cause the change in ordering of projected points on the straight line $p$ and thus the distances between neighbours may possibly take more than two values. Our generalization of Sturmian words stems in allowing any width of the strip and any projection in the cut-and-project scheme. Sequences of points obtained in the described way are called cutand-project sequences.

In our paper we use the notion of geometrical similarity. Two point sets are said to be geometrically similar, if one is an affine image of the other. Geometrical similarity is an equivalence relation. One of the main results of the present paper is the characterization of classes of this equivalence, cf. Theorem 6.3.

Our construction of cut-and-project sequences is a special case of the definition of the so-called model sets. Properties of general model sets have been extensively studied because they are suitable models for materials with long-range aperiodic order. For example, it is well known [18] that every model set has a finite number of local configurations. In particular, for our one-dimensional case it means that there are only finitely many different distances between adjacent points.

In our article we show that the distances between neighbours in a cutand-project sequence take at least two and at most three values. Similar problem was studied by Langevin in [13] as a generalization of the well known three-distance theorem [21]. The theorem is a simple consequence of our Theorem 4.1 which will be derived in the paper. 
To a cut-and-project sequence we can associate a symbolic sequence in a two- or three-letter alphabet. Obviously, geometrically similar cut-andproject sequences determine identical symbolic sequences. The opposite statement is however not true. Even two cut-and-project sequences that are not geometrically similar may provide coinciding words. It is therefore practical to introduce the notion of combinatorial equivalence. In Theorem 7.5 we describe the equivalence classes.

Another result of this paper is the description of complexity of words associated to cut-and-project sequences (Theorem 5.3). We show that the binary cut-and-project words have complexity $n+1$ and therefore are Sturmian; the ternary words correspond to codings of three-interval exchange transformations with permutation $(3,2,1)$.

\section{Words}

A finite alphabet is a set of symbols $\mathcal{A}=\left\{a_{1}, \ldots, a_{k}\right\}$. A concatenation $w$ of letters is called a word. The length of a word $w$ is the number of letters from which $w$ is formed. We denote by $\mathcal{A}^{*}$ the set of words in the alphabet $\mathcal{A}$. A one-way infinite word $u$ is a sequence $u=\left(u_{n}\right)_{n \in \mathbb{N}}=u_{1} u_{2} u_{3} \ldots$ with values in $\mathcal{A}$. One may consider also bidirectional infinite words as sequences $u=\left(u_{n}\right)_{n \in \mathbb{Z}}$. In our article we work with bidirectional infinite words. Two words $\left(u_{n}\right)_{n \in \mathbb{Z}}$ in an alphabet $\mathcal{A}$ and $\left(v_{n}\right)_{n \in \mathbb{Z}}$ in an alphabet $\mathcal{B}$ are combinatorially equivalent if there exists a bijection $h: \mathcal{A} \rightarrow \mathcal{B}$ and an $n_{0} \in \mathbb{Z}$ such that $h\left(u_{n}\right)=v_{n+n_{0}}$ for all $n \in \mathbb{Z}$.

Let $i \in \mathbb{Z}, n \in \mathbb{N}$. A concatenation $u_{i} u_{i+1} \ldots u_{i+n-1}$ is called a factor of $u$ of length $n$. We define the density of a factor $w$ of $u$ of length $n$ as

$$
\varrho_{w}=\lim _{k \rightarrow \infty} \frac{\#\left\{i \in \mathbb{Z} \cap[-k, k] \mid u_{i} u_{i+1} \ldots u_{i+n-1}=w\right\}}{2 k+1},
$$

if the limit exists.

The function that assigns to a positive integer $n$ the number of different factors of length $n$ in an infinite word $u$ is called the complexity of $u$, usually denoted by $\mathcal{C}$,

$$
\mathcal{C}(n):=\#\left\{u_{i} u_{i+1} \ldots u_{i+n-1} \mid i \in \mathbb{Z}\right\}
$$

For the complexity of an infinite word in a finite alphabet $\mathcal{A}$ one has

$$
1 \leq \mathcal{C}(n) \leq(\# \mathcal{A})^{n} \text {. }
$$

The complexity function is thus a measure of disorder in the infinite word. For more about the complexity function see $[3,7,10]$.

Let us recall some facts about one-way infinite words. We say that an infinite word $u=u_{1} u_{2} u_{3} \ldots$ in a finite alphabet $\mathcal{A}$ is eventually periodic, if there exist finite words $w_{0}, w_{1}$ in $\mathcal{A}^{*}$ such that $w=w_{0} w_{1} w_{1} w_{1} \ldots$ A word which is not eventually periodic is called aperiodic. It is well known that a one-way infinite word is eventually periodic if and only if there exists $n \in \mathbb{N}$, 
such that $\mathcal{C}(n) \leq n$, (see $[17,9])$. It follows that the most simple aperiodic one-way infinite words are those of complexity $\mathcal{C}(n)=n+1$. Such words are called Sturmian. A nice survey of Sturmian and related sequences may be found in $[17,15,14]$. Algebraically, every Sturmian word can be written as a sequence of 0 's and 1's given by one of the following formulas

$$
\begin{aligned}
& u_{n}(\alpha, \beta)=\lfloor(n+1) \alpha+\beta\rfloor-\lfloor n \alpha+\beta\rfloor, \\
& u_{n}(\alpha, \beta)=\lceil(n+1) \alpha+\beta\rceil-\lceil n \alpha+\beta\rceil,
\end{aligned}
$$

for $\alpha, \beta \in[0,1), \alpha$ irrational. Note that the above expressions can be extended for $n \in \mathbb{Z}$ so that they define aperiodic bidirectional infinite words.

J. Cassaigne defines in [8] the quasisturmian sequences as such infinite sequences for which there exist integers $k$ and $n_{0}$ such that the complexity function is $\mathcal{C}(n)=n+k$ for $n \geq n_{0}$. An example of a quasisturmian sequence is a sequence that arises from a Sturmian word if one substitutes every 0 by a finite word $w_{0}$ and every 1 by a finite word $w_{1}$. Cassaigne has shown that every quasisturmian sequence is, up to a finite prefix, of this kind.

Using the powerful notion of the Rauzy graphs, the words with complexity $2 n+1$ can be divided into four classes according to the maximal indegree and outdegree in the associated sequence of directed graphs. Arnoux and Rauzy gave geometrical characterization of infinite words of type 3-3. We show that cut-and-project sequences are geometrical reprezentations of infinite words of type 2-2.

In order to explain the classification of sequences with complexity $2 n+1$ into four groups, let us recall the definition of a Rauzy graph. A Rauzy graph for a given infinite word $u$ and given integer $n$ is an oriented graph $\Gamma_{n}=\left(V_{n}, E_{n}\right)$, where the vertices are represented by factors of length $n$ and the oriented edges are determined by factors of length $n+1$. An edge $e$ starts in a vertex $v_{1}$ and terminates in a vertex $v_{2}$ if the factor $v_{1}$ of length $n$ is a prefix of the factor $e$ and $v_{2}$ is its suffix. Thus $\# V_{n}=\mathcal{C}(n)$ and $\# E_{n}=\mathcal{C}(n+1)$. The number of edges that start (end) in a vertex $v$ is called the outdegree (indegree) of $v$. For a bidirectional infinite word, every vertex of every graph has both in- and outdegree at least one. For words of complexity $2 n+1$ in every graph $\Gamma_{n}$ the number of edges is equal to the number of vertices plus 2. Therefore the maximal outdegree for the graph is either 2 or 3 . The same statement is true for maximal indegree. The pair maximal indegree - maximal outdegree for words of complexity $2 n+1$ can therefore have only four values. This classification holds for sequences for which the indegrees are always non-zero. This is the case if the word is bidirectional or one-way recurrent. Arnoux and Rauzy in [4] show that if for some $n_{0}$ the maximal outdegree in the graph $\Gamma_{n_{0}}$ is 2 , then it is 2 for every graph $\Gamma_{n}, n \geq n_{0}$. The same statement again holds for indegree. In 
the article the authors find geometrical representation of words with Rauzy graphs of the type 3-3 for every $n \in \mathbb{N}$. The infinite words considered in this article are of the type 2-2, which means that starting from a certain $n_{0}$ both maximal indegree and maximal outdegree are equal to 2 .

\section{Cut-and-project sequences}

Generally, cut-and-project sets are defined as projections of lattices of arbitrary dimensions [18]. In this paper we study the simpliest case. The cut-and-project scheme in $\mathbb{R}^{2}$ is given by two one-dimensional subspaces $V_{1}$ and $V_{2}$ and by projections $\pi_{1}: \mathbb{R}^{2} \rightarrow V_{1}$ and $\pi_{2}: \mathbb{R}^{2} \rightarrow V_{2}$ which satisfy:

1) $\pi_{1}$ restricted to $\mathbb{Z}^{2}$ is an injection.

2) $\pi_{2}\left(\mathbb{Z}^{2}\right)$ is dense in $V_{2}$.

The scheme is illustrated by the following picture.

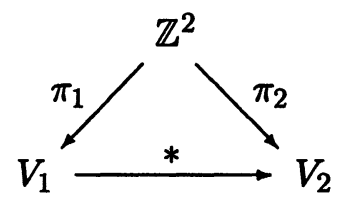

In this scheme $\pi_{1}\left(\mathbb{Z}^{2}\right)$ and $\pi_{2}\left(\mathbb{Z}^{2}\right)$ are additive abelian groups. The bijection between them, $\pi_{1}^{-1} \circ \pi_{2}$ is usually denoted by $*$ and called the star map. Its inverse is denoted by $-*$.

Let $V_{1}$ be the linear span of a vector $\overrightarrow{x_{1}}=(1, \varepsilon)$ and $V_{2}$ the linear span of a vector $\overrightarrow{x_{2}}=(-1, \eta)$. In order to satisfy conditions 1$)$ and 2$)$ we choose $\varepsilon, \eta$ irrational and $\varepsilon \neq-\eta$. Any vector $(p, q) \in \mathbb{Z}^{2}$ can be written as

$$
(p, q)=\frac{1}{\varepsilon+\eta}(q+\eta p) \vec{x}_{1}+\frac{1}{\varepsilon+\eta}(q-\varepsilon p) \vec{x}_{2}=\pi_{1}(p, q)+\pi_{2}(p, q) .
$$

For simplicity we omit the common factor $1 /(\varepsilon+\eta)$ (which corresponds to different normalization of vectors $\overrightarrow{x_{1}}$ and $\overrightarrow{x_{2}}$ ) and consider the abelian groups

$$
\mathbb{Z}[\eta]=\{p+q \eta \mid p, q \in \mathbb{Z}\} \quad \text { and } \quad \mathbb{Z}[\varepsilon]=\{p+q \varepsilon \mid p, q \in \mathbb{Z}\} .
$$

The star map $*: \mathbb{Z}[\eta] \rightarrow \mathbb{Z}[\varepsilon]$, given by

$$
x=p+q \eta \quad \mapsto \quad x^{*}=p-q \varepsilon,
$$

is an isomorphism of the two groups. Using this formalism we can easily define a cut-and-project sequence as

$$
\Sigma_{\varepsilon, \eta}(\Omega)=\left\{x \in \mathbb{Z}[\eta] \mid x^{*} \in \Omega\right\},
$$

where $\Omega$ is a bounded interval, called the acceptance window. We denote the length of an interval $\Omega$ by $|\Omega|$. An example of the construction of a cut-and-project sequence is found on Figure 1. 


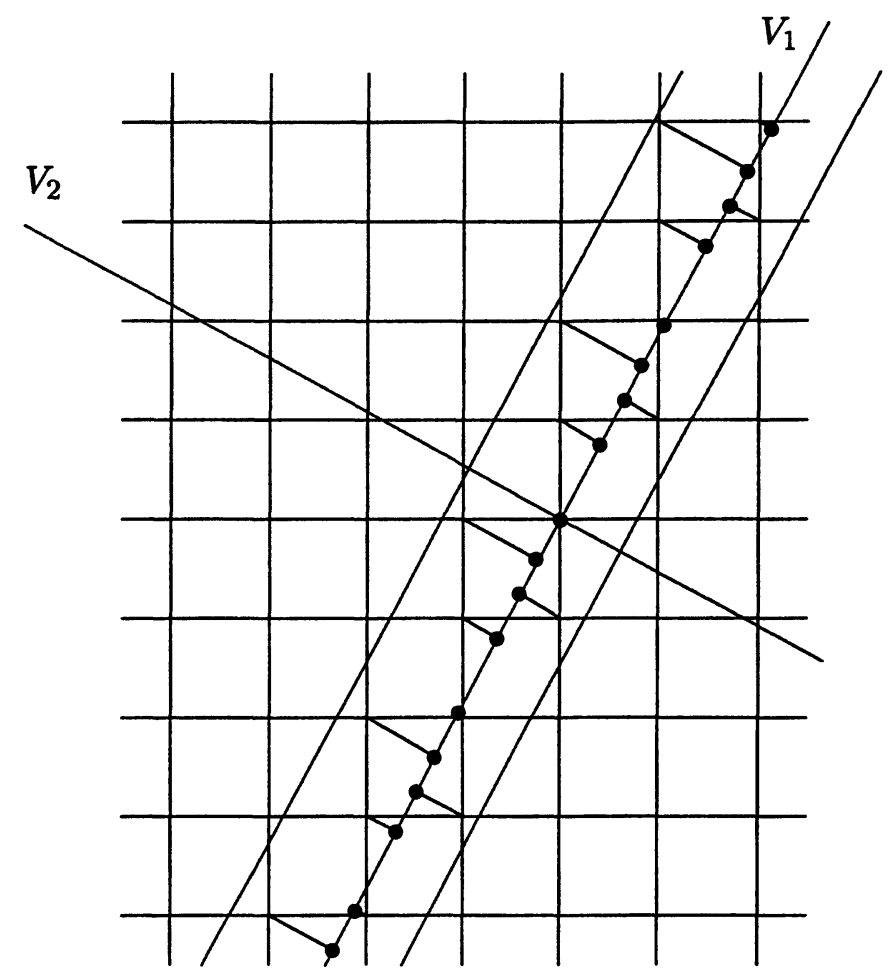

Figure 1. Construction of a cut-and-project sequence.

It is well known [18] that any cut-and-project set is Delone with finite number of polygons in Voronoi tiling of space. For $\Sigma_{\varepsilon, \eta}(\Omega) \subset \mathbb{R}$ it implies that there exists an increasing sequence $\left(x_{n}\right)_{n \in \mathbb{Z}}$ such that $\Sigma_{\varepsilon, \eta}(\Omega)=$ $\left\{x_{n} \mid n \in \mathbb{Z}\right\}$ and the set of tiles $T=\left\{t_{n}=x_{n+1}-x_{n} \mid n \in \mathbb{Z}\right\}$ is finite.

If we assign to each tile $t \in T$ a letter $h(t)$ from an alphabet $\mathcal{A}$, the sequence $\left(h\left(t_{n}\right)\right)_{n \in \mathbb{Z}}$ can thus be viewed as a bidirectional infinite word in the finite alphabet $\mathcal{A}$.

For the description of $\Sigma_{\varepsilon, \eta}(\Omega)$ we can use a different increasing sequence $\tilde{x}_{n}:=x_{n+p}$ for arbitrary fixed $p \in \mathbb{Z}$. The corresponding sequence $\left(\tilde{t}_{n}\right)_{n \in \mathbb{Z}}$ has then the property $\tilde{t}_{n}=t_{n+p}$ for all $n \in \mathbb{Z}$. The word $\left(h\left(\tilde{t}_{n}\right)\right)_{n \in \mathbb{Z}}$ is thus a $p$-shift of the infinite word $\left(h\left(t_{n}\right)\right)_{n \in \mathbb{Z}}$. In order to avoid specification of the point indexed by 0 , we introduce the symbol $u_{\varepsilon, \eta}(\Omega)$ for the entire class of all bidirectional infinite words, that are associated to $\Sigma_{\varepsilon, \eta}(\Omega)$. In [17] the infinite bidirectional words without specific origin are called trajectories. The terminology pointed/non-pointed words is also used.

We are interested in combinatorial properties of words $u_{\varepsilon, \eta}(\Omega)$. In particular, we study 
- the cardinality of the alphabet $\{h(t) \mid t \in T\} \subseteq \mathcal{A}$, in case when different tiles are assigned to different letters, i.e., the map $h$ is injective,

- the subword complexity of $u_{\varepsilon, \eta}(\Omega)$,

- the triples of parameters $\varepsilon, \eta, \Omega$ that give the same bidirectional infinite words, up to the choice of the letter assignment $h$ into the alphabet $\mathcal{A}$.

\section{Distances in $\Sigma_{\varepsilon, \eta}(\Omega)$}

In this section we first determine the number of different tiles in $\Sigma_{\varepsilon, \eta}(\Omega)$ and then we derive the lengths of these tiles.

Let us first focus on cut-and-project sequences with symmetric acceptance interval $\Omega=(-d, d)$, with $d>0$. Let $0<x_{1}<x_{2}<\cdots$ denote the positive elements of the set $\Sigma_{\varepsilon, \eta}(-d, d)$. Let $x_{k}$ be the minimal positive element of $\Sigma_{\varepsilon, \eta}(-d, d)$ with sign $x_{k}^{*} \neq \operatorname{sign} x_{1}^{*}$. Since the images $x_{n}^{*}$ cover densely the interval $(-d, d)$, such an $x_{k}$ must exist. Then $0<x_{i}-x_{1}<x_{i}<x_{k}$ for all $i=2,3, \ldots, k-1$ and $\left(x_{i}-x_{1}\right)^{*} \in(-d, d)$. Thus $x_{i}-x_{1}$ are positive elements of $\Sigma_{\varepsilon, \eta}(-d, d)$ which is possible only as $x_{i}-x_{1}=x_{j}$ for some $j=1,2, \ldots, i-1$. This implies that

$$
x_{i}=i x_{1}, \quad \text { for } i=1,2, \ldots, k-1 .
$$

Moreover,

$$
\left|x_{k}^{*}-x_{1}^{*}\right| \geq d .
$$

Indeed, suppose that $x_{k}^{*}-x_{1}^{*}=\left(x_{k}-x_{1}\right)^{*} \in(-d, d)$, i.e., $x_{k}-x_{1} \in$ $\Sigma_{\varepsilon, \eta}(-d, d)$. Since $x_{k}>x_{k}-x_{1}>0$, according to (2) there must exist a $j \in\{0,1, \ldots, k-1\}$, such that $x_{k}-x_{1}=j x_{1}$, which gives $x_{k}^{*}=(j+1) x_{1}^{*}$. The latter is a contradiction with the assumption $\operatorname{sign} x_{k}^{*} \neq \operatorname{sign} x_{1}^{*}$.

Theorem 4.1. For any irrational numbers $\varepsilon, \eta, \varepsilon \neq-\eta$, and any bounded interval $\Omega=[c, c+d)$, there exist positive numbers $\Delta_{1}$ and $\Delta_{2}$ such that the distances between consecutive points in $\Sigma_{\varepsilon, \eta}(\Omega)$ take at most three values among $\left\{\Delta_{1}, \Delta_{2}, \Delta_{1}+\Delta_{2}\right\}$.

Proof. The points of $\Sigma_{\varepsilon, \eta}[c, c+d)$ form an increasing sequence $\left(x_{n}\right)_{n \in \mathbb{Z}}$, i.e., $\Sigma_{\varepsilon, \eta}[c, c+d)=\left\{x_{n} \mid n \in \mathbb{Z}\right\}$. A positive number $\Delta$ is a distance between consecutive points of $\Sigma_{\varepsilon, \eta}[c, c+d)$ if there exists $n \in \mathbb{Z}$, such that $\Delta=x_{n+1}-x_{n}$. For any tile $\Delta>0$ we have $\Delta \in \Sigma_{\varepsilon, \eta}(-d, d)$. Moreover, let both $\delta>0$ and $i \delta, i \in \mathbb{N}, i \geq 2$, belong to $\Sigma_{\varepsilon, \eta}(-d, d)$. Then $i \delta$ is not a tile in $\Sigma_{\varepsilon, \eta}[c, c+d)$, since if $x$ and $x+i \delta$ are elements of $\Sigma_{\varepsilon, \eta}[c, c+d)$ then also $x+\delta$ belongs to $\Sigma_{\varepsilon, \eta}[c, c+d)$, and hence $x+i \delta$ is not the closest neighbour of $x$.

Denote by $\Delta_{1}$ the smallest positive element of $\Sigma_{\varepsilon, \eta}(-d, d)$ with $\Delta_{1}^{*}>0$, and denote by $\Delta_{2}$ the smallest positive element of $\Sigma_{\varepsilon, \eta}(-d, d)$ satisfying 
$\Delta_{2}^{*}<0$. Formally,

$$
\begin{aligned}
& \Delta_{1}=\min \left\{x>0 \mid x \in \Sigma_{\varepsilon, \eta}(0, d)\right\}, \\
& \Delta_{2}=\min \left\{x>0 \mid x \in \Sigma_{\varepsilon, \eta}(-d, 0)\right\} .
\end{aligned}
$$

The values $\Delta_{1}$ and $\Delta_{2}$ are the two smallest candidates to be lengths of tiles in $\Sigma_{\varepsilon, \eta}[c, c+d)$.

According to (3) we have

$$
\Delta_{1}^{*}-\Delta_{2}^{*} \geq d
$$

Let $x_{1} \in \Sigma_{\varepsilon, \eta}[c, c+d)$ such that $x_{1}^{*} \in\left[c, c+d-\Delta_{1}^{*}\right)$. Since $x_{1}^{*}+\Delta_{1}^{*} \in$ $[c, c+d)$ and $x_{1}^{*}+\Delta_{2}^{*}<c+d-\Delta_{1}^{*}+\Delta_{2}^{*} \leq c$ (compare (4)), the point $x_{1}+\Delta_{1}$ belongs to $\Sigma_{\varepsilon, \eta}[c, c+d)$ and $x_{1}+\Delta_{1}$ is the closest right neighbour of $x_{1}$.

Let $x_{2} \in \Sigma_{\varepsilon, \eta}[c, c+d)$ such that $x_{2}^{*} \in\left[c-\Delta_{2}^{*}, c+d\right)$. Similarly, $x_{2}^{*}+\Delta_{2}^{*} \in$ $[c, c+d)$ and $x_{2}^{*}+\Delta_{1}^{*} \notin[c, c+d)$, therefore $x_{2}+\Delta_{2}$ is the closest right neighbour of $x_{2}$.

Now let $x_{3} \in \Sigma_{\varepsilon, \eta}[c, c+d)$ such that $x_{3}^{*} \in\left[c+d-\Delta_{1}^{*}, c-\Delta_{2}^{*}\right)$. Then

$$
c \leq c+d+\Delta_{2}^{*} \leq x_{3}^{*}+\Delta_{1}^{*}+\Delta_{2}^{*}<c+\Delta_{1}^{*}<c+d,
$$

and the point $x_{3}+\Delta_{1}+\Delta_{2}$ belongs to $\Sigma_{\varepsilon, \eta}[c, c+d)$. We want to show that $x_{3}+\Delta_{1}+\Delta_{2}$ is the closest right neighbour of the point $x_{3}$.

Suppose that there exists a tile $\Delta$, such that

$$
x_{3}<x_{3}+\Delta<x_{3}+\Delta_{1}+\Delta_{2}, \quad x_{3}+\Delta \in \Sigma_{\varepsilon, \eta}[c, c+d) .
$$

Combining the inequalities

$$
c+d-\Delta_{1}^{*} \leq x_{3}^{*}<c-\Delta_{2}^{*}, \text { and } c \leq x_{3}^{*}+\Delta^{*}<c+d
$$

we obtain

$$
\Delta_{2}^{*}<\Delta^{*}<\Delta_{1}^{*} .
$$

Consider the point $\delta:=\Delta_{1}+\Delta_{2}-\Delta>0$. Since $\Delta>\Delta_{1}, \Delta_{2}$ (as $\Delta_{1}, \Delta_{2}$ are the two smallest candidates for lengths of tiles), it holds that $0<\delta<$ $\min \left\{\Delta_{1}, \Delta_{2}\right\}$ but using (5) we get

$$
-d<\Delta_{2}^{*}<\delta^{*}=\Delta_{1}^{*}+\Delta_{2}^{*}-\Delta^{*}<\Delta_{1}^{*}<d .
$$

It means that $\delta \in \Sigma_{\varepsilon, \eta}(-d, d)$, which contradicts the definition of $\Delta_{1}$ and $\Delta_{2}$ as being the smallest elements of $\Sigma_{\varepsilon, \eta}(-d, d)$. As a result, the closest right neighbour of $x_{3} \in \Sigma_{\varepsilon, \eta}[c, c+d)$, with $x_{3}^{*} \in\left[c+d-\Delta_{1}^{*}, c-\Delta_{2}^{*}\right)$, is the point $x_{3}+\Delta_{1}+\Delta_{2}$.

By that we have determined the right neighbours of all the elements of $\Sigma_{\varepsilon, \eta}[c, c+d)$ and the proof is completed.

Let us make several comments on the above theorem: 
- From the proof of the above theorem it follows that $\Delta_{1}$ and $\Delta_{2}$ depend only on the length $d$ of the interval $\Omega=[c, c+d)$ and not on the position of the point $c$. The lengths of tiles $\Delta_{1}$ and $\Delta_{2}$ satisfy $\Delta_{1}^{*} \Delta_{2}^{*}<0$ and we shall always denote by $\Delta_{1}$ the tile with positive star image $\Delta_{1}^{*}>0$, similarly, $\Delta_{2}^{*}<0$.

- If the acceptance window were an interval $\Omega=(c, c+d]$ or $\Omega=$ $(c, c+d)$ the proof of Theorem 4.1 could be repeated identicaly; for the case $\Omega=[c, c+d]$ a minor amendment is needed. Hence for every acceptance interval $\Omega$ with non-empty interior the set $\Sigma_{\varepsilon, \eta}(\Omega)$ has at most three types of tiles.

- Theorem 4.1 implies that every segment of the set $\Sigma_{\varepsilon, \eta}(\Omega)$ has three types of distances $\Delta_{1}, \Delta_{2}, \Delta_{1}+\Delta_{2}$ between neighbouring points. More precisely, the set

$$
J \cap \Sigma_{\varepsilon, \eta}(\Omega)=\{a+b \eta \in J \mid a, b \in \mathbb{Z}, a-b \varepsilon \in \Omega\}
$$

has at most three distances between neighbours for arbitrary irrational $\varepsilon, \eta, \varepsilon \neq-\eta$, and arbitrary bounded intervals $J, \Omega$. For a given positive irrational $\alpha$ and a positive integer $n$ we define $\eta=-\alpha$, $\varepsilon=-\frac{1}{\alpha}, J=(0,1]$, and $\Omega=[1, K)$, where $K=[\alpha(n+1)]+$ $\frac{1}{\alpha}(n+1)+1$. Then

$$
M=\left\{a-b \alpha \in(0,1] \mid a, b \in \mathbb{Z}, a+\frac{b}{\alpha} \in[1, K)\right\} .
$$

The condition $0<a-b \alpha \leq 1$ implies $a=1+[b \alpha]$, hence $a-b \alpha=$ $1-\{b \alpha\}$. We also have

$$
a+\frac{b}{\alpha}=1+[b \alpha]+\frac{b}{\alpha} \in[1, K) \quad \Leftrightarrow \quad 0 \leq b<n+1 .
$$

We thus obtain $M=\{1-\{k \alpha\} \mid k=0,1, \ldots, n\}$. The set $M$ has at most three distances between neighbours. The same property is satisfied by $1-M=\{\{k \alpha\} \mid k=0,1, \ldots, n\}$, which is the well known three-distance theorem [21].

From the proof of Theorem 4.1 we may derive a prescription to find the right neighbour of a given point $x$ in the cut-and-project sequence, according to the position of $x^{*}$ in the acceptance interval.

Corollary 4.2. Let $\Omega=[c, c+d)$ be a bounded interval, and let $\Delta_{1}, \Delta_{2}$, and $\Delta_{1}+\Delta_{2}$ be the tiles in $\Sigma_{\varepsilon, \eta}(\Omega)$ such that $\Delta_{1}^{*}>0>\Delta_{2}^{*}$. The closest right neighbour of a point $x \in \Sigma_{\varepsilon, \eta}(\Omega)$ is

$$
\begin{array}{ll}
x+\Delta_{1} & \text { if } x^{*} \in\left[c, c+d-\Delta_{1}^{*}\right), \\
x+\Delta_{1}+\Delta_{2} & \text { if } x^{*} \in\left[c+d-\Delta_{1}^{*}, c-\Delta_{2}^{*}\right), \\
x+\Delta_{2} & \text { if } x^{*} \in\left[c-\Delta_{2}^{*}, c+d\right) .
\end{array}
$$


In the remaining part of this section we derive the lengths of tiles in $\Sigma_{\varepsilon, \eta}(\Omega)$ for a given interval $\Omega=[c, c+d)$ and positive $\varepsilon, \eta$. As it will be seen from the study of geometrical similarities of cut-and-project sets, the assumption of $\varepsilon, \eta$ positive does not cause a loss of generality.

Changing continuously the length $d$ of the acceptance interval $\Omega=[c, c+d)$ causes discrete changes of the triplet of distances $\left(\Delta_{1}, \Delta_{2}\right.$, $\left.\Delta_{1}+\Delta_{2}\right)$. Let $\Sigma_{\varepsilon, \eta}[c, c+d)$ be a generic cut-and-project sequence, and let $\Delta_{1}^{*}>0, \Delta_{2}^{*}<0$ and $\Delta_{1}^{*}+\Delta_{2}^{*}$ be the star map images of its tiles.

Denote by $d_{+}$the smallest number such that $d<d_{+}$and at least one of the distances $\Delta_{1}, \Delta_{2}, \Delta_{1}+\Delta_{2}$ does not occur in $\Sigma_{\varepsilon, \eta}\left[c, c+d_{+}\right)$. Similarly, denote by $d_{-}$the largest number such that $d_{-}<d$ and at least one of the distances $\Delta_{1}, \Delta_{2}, \Delta_{1}+\Delta_{2}$ does not occur in $\Sigma_{\varepsilon, \eta}\left[c, c+d_{-}\right)$. Due to Corollary 4.2, we have $d_{+}=\Delta_{1}^{*}-\Delta_{2}^{*}$ and thus $\Sigma_{\varepsilon, \eta}\left[c, c+d_{+}\right)$has only two distances, namely $\Delta_{1}$ and $\Delta_{2}$. Hence growing the length of the acceptance interval, the largest distance $\Delta_{1}+\Delta_{2}$ disappears.

Similar situation occurs in $\Sigma_{\varepsilon, \eta}\left[c, c+d_{-}\right)$. One of the distances $\Delta_{1}$, $\Delta_{2}, \Delta_{1}+\Delta_{2}$ disappears, but it happens in such a way that the remaining distances have their star map images of opposite sign. Thus $\Sigma_{\varepsilon, \eta}\left[c, c+d_{-}\right)$ has distances

$$
\begin{array}{ll}
\Delta_{1}, \Delta_{1}+\Delta_{2} & \text { if } \Delta_{1}^{*}+\Delta_{2}^{*}<0 \\
\Delta_{2}, \Delta_{1}+\Delta_{2} & \text { if } \quad \Delta_{1}^{*}+\Delta_{2}^{*}>0
\end{array}
$$

From Corollary 4.2 we obtain

$$
d_{-}=\Delta_{1}^{*}-\left(\Delta_{1}+\Delta_{2}\right)^{*}=-\Delta_{2}^{*}, \quad \text { or } \quad d_{-}=\Delta_{1}^{*},
$$

respectively.

Starting from a given initial value $d_{0}$, for which the set $\Sigma_{\varepsilon, \eta}\left[c, c+d_{0}\right)$ has 2 tiles, we can determine by recurrence all lengths $d_{n}, n \in \mathbb{Z}$, of the acceptance windows for which $\Sigma_{\varepsilon, \eta}\left[c, c+d_{n}\right)$ has only two tiles. The initial value $d_{0}$ is determined below in Example 1 .

Let $\Delta_{n 1}^{*}>0$ and $\Delta_{n 2}^{*}<0$ be the star images of distances occurring in the sequence $\Sigma_{\varepsilon, \eta}\left[0, d_{n}\right)$, i.e., $d_{n}=\Delta_{n 1}^{*}-\Delta_{n 2}^{*}$.

(6)

$$
\begin{aligned}
& \text { If } \Delta_{n 1}^{*}+\Delta_{n 2}^{*}>0 \text { then } \begin{cases}d_{n+1} & :=\Delta_{n 1}^{*}, \\
\Delta_{(n+1) 1}^{*} & :=\Delta_{n 1}^{*}+\Delta_{n 2}^{*}, \\
\Delta_{(n+1) 2}^{*} & :=\Delta_{n 2}^{*}\end{cases} \\
& \text { If } \Delta_{n 1}^{*}+\Delta_{n 2}^{*}<0 \text { then }\left\{\begin{array}{lll}
d_{n+1} & :=-\Delta_{n 2}^{*}, \\
\Delta_{(n+1) 1}^{*} & :=\Delta_{n 1}^{*}, \\
\Delta_{(n+1) 2}^{*} & :=\Delta_{n 1}^{*}+\Delta_{n 2}^{*} .
\end{array}\right.
\end{aligned}
$$


Similarly, the algorithm which from the triple $d_{n}, \Delta_{n 1}, \Delta_{n 2}$ finds the triple $d_{n-1}, \Delta_{(n-1) 1}, \Delta_{(n-1) 2}$ has the inverse form

$$
\begin{aligned}
\text { If } \Delta_{n 1}>\Delta_{n 2} \text { then } \begin{cases}d_{n-1} & :=\Delta_{n 1}^{*}-2 \Delta_{n 2}^{*}, \\
\Delta_{(n-1) 1}^{*} & :=\Delta_{n 1}^{*}-\Delta_{n 2}^{*}, \\
\Delta_{(n-1) 2}^{*} & :=\Delta_{n 2}^{*}\end{cases} \\
\text { If } \Delta_{n 1}<\Delta_{n 2} \text { then } \begin{cases}d_{n-1}^{*} & :=2 \Delta_{n 1}^{*}-\Delta_{n 2}^{*}, \\
\Delta_{(n-1) 1}^{*} & :=\Delta_{n 1}^{*}, \\
\Delta_{(n-1) 2}^{*} & :=\Delta_{n 2}^{*}-\Delta_{n 1}^{*} .\end{cases}
\end{aligned}
$$

We have yet to determine some initial values for the recurrences (6) and (7). It turns out that it is reasonable to start with a cut-and-project sequence whose acceptance interval is of unit length. Since the distances do not depend on the position of the interval, we can focus on the acceptance interval $[0,1)$.

Example 1. Let $\varepsilon, \eta$ be fixed positive irrational numbers. Let us study the sequence $\Sigma_{\varepsilon, \eta}[0,1)=\{p+q \eta \mid p, q \in \mathbb{Z}, 0 \leq p-q \varepsilon<1\}$. Elements of this set have to satisfy $q \varepsilon \leq p<1+q \varepsilon$. Therefore we can write

$$
\Sigma_{\varepsilon, \eta}[0,1)=\left\{x_{q}:=\lceil q \varepsilon\rceil+q \eta \mid q \in \mathbb{Z}\right\} .
$$

From the above formula one may easily observe that the tiles in a $\Sigma_{\varepsilon, \eta}[0,1)$ form a Sturmian word with slope $\varepsilon$. Indeed, since $\left(x_{q}\right)_{q \in \mathbb{Z}}$ is an increasing sequence, the lengths of tiles in $\Sigma_{\varepsilon, \eta}[0,1)$ can be computed as

$$
x_{q+1}-x_{q}=\lceil q \varepsilon+\varepsilon\rceil-\lceil q \varepsilon\rceil+\eta= \begin{cases}{[\varepsilon]+1+\eta} & =: \Delta_{1} \\ {[\varepsilon]+\eta} & =: \Delta_{2}\end{cases}
$$

The sequence $\Sigma_{\varepsilon, \eta}[0,1)$ has thus two distances ordered as a Sturmian word with slope $\alpha=\varepsilon$ and shift intercept $\beta=0$, cf. definition of Sturmian words (1). We denote the length of the acceptance interval by $d_{0}=1$. In the notation of the recurrence relations (6) there is

$$
\begin{array}{ll}
\Delta_{01}^{*}=[\varepsilon]+1-\varepsilon=1-\{\varepsilon\}, & d_{0}=1, \\
\Delta_{02}^{*}=[\varepsilon]-\varepsilon=-\{\varepsilon\}, & \text { and } \quad \\
d_{1}=\max (1-\{\varepsilon\},\{\varepsilon\}) .
\end{array}
$$

It is now obvious that for the determination of the lengths of tiles in a generic cut-and-project sequence $\Sigma_{\varepsilon, \eta}[c, c+d)$ with $\varepsilon, \eta, d>0$, it suffices to find $n \in \mathbb{Z}$ such that $d_{n+1}<d<d_{n}$. Then numbers $\Delta_{n 1}, \Delta_{n 2}$, $\Delta_{(n+1) 1}, \Delta_{(n+1) 2}$ take three different values that are the lengths of tiles in $\Sigma_{\varepsilon, \eta}[c, c+d)$.

Proposition 4.3. Let $\varepsilon, \eta>0$ be irrational numbers and let $\Omega=[c, c+d)$ satisfy

$$
d_{1}=\max (1-\{\varepsilon\},\{\varepsilon\})<d<d_{0}=1 .
$$


The sequence $\Sigma_{\varepsilon, \eta}(\Omega)$ has three types of tiles and the lengths of these tiles are

$\Delta_{1}=[\varepsilon]+1+\eta, \quad \Delta_{2}=[\varepsilon]+\eta, \quad$ and $\quad \Delta_{1}+\Delta_{2}=2[\varepsilon]+1+2 \eta$.

Let us realize that in this case $\Delta_{1}^{*}$ and $\Delta_{2}^{*}$ do not depend on $\eta>0$. Therefore also the algorithm (6), corresponding to shortening the acceptance interval, does not depend on $\eta$ (unlike the algorithm (7)). The value of $\eta$ influences only the length of tiles and not their ordering. Therefore we can choose the parameter $\eta>0$ according to our needs, for example we can set $\eta=\varepsilon^{-1}$ which corresponds to the case when the projection in the cut-and-project scheme is orthogonal.

Proposition 4.4. Let $\varepsilon, \eta>0$ and let $\Omega$ be an interval of length $d$, $0<d \leq 1$. Then

$$
u_{\varepsilon, \eta}(\Omega)=u_{\varepsilon, 1 / \varepsilon}(\Omega) .
$$

In case that the acceptance window has length $d>1$, the parameter $\eta$ plays an important role, as it is shown by the following assertion, which summarizes the results of this section. Its proof is a consequence of algorithms (6) and (7) and uses mathematical induction.

Proposition 4.5. Let $\varepsilon, \eta>0$ be irrational numbers with continued fraction $\left[a_{0}, a_{1}, a_{2}, \ldots\right]$ and $\left[b_{0}, b_{1}, b_{2}, \ldots\right]$ respectively. Denote by $\left(\frac{p_{k}}{q_{k}}\right)_{k \in \mathbb{N}}$ and $\left(\frac{r_{k}}{t_{k}}\right)_{k \in \mathbb{N}}$ the sequences of the convergents associated to $\varepsilon$ and $\eta$, that is

$$
\frac{p_{k}}{q_{k}}=\left[a_{0}, a_{1}, \ldots, a_{k}\right], \quad \frac{r_{k}}{t_{k}}=\left[b_{0}, b_{1}, \ldots, b_{k}\right] .
$$

- Let $0<d<1 . \Sigma_{\varepsilon, \eta}[c, c+d)$ is a cut-and-project sequence with two tiles iff there exist $k \in \mathbb{N}_{0}$, and $s \in \mathbb{N}, 1 \leq s \leq a_{k+1}$, such that $d=\delta_{k, s}=\left|(s-1)\left(p_{k}-\varepsilon q_{k}\right)+p_{k-1}-\varepsilon q_{k-1}\right|$. In this case the lengths of tiles are

$$
p_{k}+\eta q_{k} \quad \text { and } \quad s\left(p_{k}+\eta q_{k}\right)+p_{k-1}+\eta q_{k-1} .
$$

- Let $1<d . \Sigma_{\varepsilon, \eta}[c, c+d)$ is a cut-and-project sequence with two tiles iff there exist $k \in \mathbb{N}_{0}$, and $s \in \mathbb{N}, 1 \leq s \leq b_{k+1}$, such that $d=\rho_{k, s}=$ $(s+1)\left(r_{k}+\varepsilon t_{k}\right)+r_{k-1}+\varepsilon t_{k-1}$. In this case the lengths of tiles are

$$
\left|r_{k}-\eta t_{k}\right| \quad \text { and } \quad\left|s\left(r_{k}-\eta t_{k}\right)+r_{k-1}-\eta t_{k-1}\right| .
$$

Note that we have described all acceptance windows $\Omega$, for which $\Sigma_{\varepsilon, \eta}(\Omega)$ has only two types of distances between neighbouring points. This happens if the length of $\Omega$ belongs to one of two sequences of values, $\delta_{k, s}>1$, $0<\rho_{k, s}<1$ from the above proposition. We have

$$
\begin{aligned}
\cdots<\delta_{k+1, a_{k+2}} & <\ldots<\delta_{k+1,1}<\delta_{k, a_{k+1}}<\ldots \\
\cdots & <\delta_{k, 1}<\delta_{k-1, a_{k}}<\cdots
\end{aligned}
$$


$\lim _{k \rightarrow \infty} \delta_{k, s}=0$, and the lengths of corresponding tiles increase to $\infty$. For the other sequence we have

$$
\begin{aligned}
\cdots<\rho_{k-1,1} & <\ldots<\rho_{k-1, a_{k}}<\rho_{k, 1}<\ldots \\
\cdots & <\rho_{k, a_{k+1}}<\rho_{k+1,1}<\cdots<\rho_{k+1, a_{k+2}}<\ldots,
\end{aligned}
$$

$\lim _{k \rightarrow \infty} \rho_{k, s}=\infty$, and the lengths of corresponding tiles tend to 0 .

\section{Complexity of cut-and-project sequences}

It is useful to introduce a function that allows to determine the neighbour of a point $x \in \Sigma_{\varepsilon, \eta}(\Omega)$ according to the position of $x^{*}$ in $\Omega$. Its definition is based on Corollary 4.2 .

Definition 5.1. Let $\Omega=[c, c+d)$ be a bounded interval, and let $\Delta_{1}, \Delta_{2}$, and $\Delta_{1}+\Delta_{2}$ be the tiles in $\Sigma_{\varepsilon, \eta}(\Omega)$, such that $\Delta_{1}^{*}>0>\Delta_{2}^{*}$. Let

$$
f_{\varepsilon, \Omega}(y):= \begin{cases}y+\Delta_{1}^{*} & \text { for } y \in\left[c, c+d-\Delta_{1}^{*}\right), \\ y+\Delta_{1}^{*}+\Delta_{2}^{*} & \text { for } y \in\left[c+d-\Delta_{1}^{*}, c-\Delta_{2}^{*}\right), \\ y+\Delta_{2}^{*} & \text { for } y \in\left[c-\Delta_{2}^{*}, c+d\right) .\end{cases}
$$

The function $f_{\varepsilon, \Omega}$ is called the stepping function of $\Sigma_{\varepsilon, \eta}(\Omega)$. If there is no misunderstanding possible, we shall omit the subscripts.

The map $f$ is actually an exchange of three intervals. Codings of threeinterval exchange transformations are studied for instance in [11]. The three-interval exchange corresponding to the map $f$ from the above definition is associated with the permutation $(3,2,1)$. Every exchange of three intervals with permutation $(3,2,1)$ can be realized as a map $f_{\varepsilon, \Omega}$.

Note that the stepping function is obviously invertible. The right neighbour of $x \in \Sigma_{\varepsilon, \eta}(\Omega)$ is $\left(f_{\varepsilon, \Omega}\left(x^{*}\right)\right)^{-*}$. Similarly, the $n$-tuple of its right neighbours is given by $\left(f\left(x^{*}\right)\right)^{-*},\left(f^{(2)}\left(x^{*}\right)\right)^{-*}, \ldots,\left(f^{(n)}\left(x^{*}\right)\right)^{-*}$. In particular, for any given $y_{0} \in \Omega \cap \mathbb{Z}[\varepsilon]$ we have

$$
\Sigma_{\varepsilon, \eta}(\Omega)=\left\{\left(f_{\varepsilon, \Omega}^{(n)}\left(y_{0}\right)\right)^{-*} \mid n \in \mathbb{Z}\right\} .
$$

Let us denote the tile $\Delta_{1}+\Delta_{2}$ by letter $A$, the tile $\Delta_{1}$ by letter $\mathrm{B}$ and the tile $\Delta_{2}$ by letter $C$. According to the first $n$ right neighbours, we can associate to every point $x \in \Sigma_{\varepsilon, \eta}(\Omega)$ a word of length $n$ in the alphabet $\mathcal{A}=\{A, B, C\}$. This word will be denoted by $\operatorname{word}(x, n)$. For $x_{1}^{*}, x_{2}^{*} \in \Omega \cup \mathbb{Z}[\varepsilon]$ the words $\operatorname{word}\left(x_{1}, n\right)$ and $\operatorname{word}\left(x_{2}, n\right)$ are different if and only if there exists an $i=1,2, \ldots, n$, such that at least one discontinuity point of $f$ lies between $f^{(i-1)}\left(x_{1}^{*}\right)$ and $f^{(i-1)}\left(x_{2}^{*}\right)$. Therefore the set of all points $x^{*} \in \Omega \cap \mathbb{Z}[\varepsilon]$, satisfying $\operatorname{word}(x, n)=w$, has the form $I \cap \mathbb{Z}[\varepsilon]$ where $I$ is an interval. Such interval corresponding to the word $w$ will be denoted 
by $\Omega_{w}$ and without loss of generality we shall consider $\Omega$ semi-closed. In particular, we have

$\Omega_{A}=\left[c+d-\Delta_{1}^{*}, c-\Delta_{2}^{*}\right), \quad \Omega_{B}=\left[c, c+d-\Delta_{1}^{*}\right), \quad \Omega_{C}=\left[c-\Delta_{2}^{*}, c+d\right)$.

It is known that the density of points in a cut-and-project set is proportional to the volume of the acceptance window [19]. It follows that the density of the word $w$ is proportional to the length of interval $\Omega_{w}$. In particular, we have the following proposition.

Proposition 5.2. Let $\varepsilon, \eta$ be irrational numbers, $\varepsilon \neq-\eta$, and let $\Omega$ be a bounded interval. Let $w$ be a factor in the word $u_{\varepsilon, \eta}(\Omega)$. Then for the density $\varrho_{w}$ of the factor $w$ we have

$$
\varrho_{w}=\frac{\left|\Omega_{w}\right|}{|\Omega|} .
$$

In particular, for the densities of letters $A, B, C$ we have

$$
\varrho_{A}=\frac{\Delta_{1}^{*}-\Delta_{2}^{*}-|\Omega|}{|\Omega|}, \quad \varrho_{B}=\frac{|\Omega|-\Delta_{1}^{*}}{|\Omega|}, \quad \varrho_{C}=\frac{|\Omega|+\Delta_{2}^{*}}{|\Omega|} .
$$

Recall that the complexity $\mathcal{C}(n)$ of an infinite word $u$ is the number of different factors in $u$ of length $n$. In our case it is given by the number of all non-empty intervals $\Omega_{w}$ that cover $\Omega$. The number of such intervals is determined from the number of left end-points of these intervals. A point $z$ is a left end-point of a non-empty interval $\Omega_{w}$ if and only if it is either a left end-point of $\Omega$ itself, i.e., $z=c$, or there exists $i \in\{0,1,2 \ldots n-1\}$ such that $f^{i}(z)$ is a discontinuity point of the function $f$, i.e., $f^{i}(z) \in$ $\left\{c+d-\Delta_{1}^{*}, c-\Delta_{2}^{*}\right\}$. Therefore we have the following prescription for the complexity,

$$
\mathcal{C}(n)=\#\left\{c, \alpha, f^{(-1)}(\alpha), \ldots, f^{(-(n-1))}(\alpha), \beta, f^{(-1)}(\beta), \ldots, f^{(-(n-1))}(\beta)\right\}
$$

where we denote for simplicity of notation $\alpha=c+d-\Delta_{1}^{*}$ and $\beta=c-\Delta_{2}^{*}$.

We can now determine the complexity of $u_{\varepsilon, \eta}[c, c+d)$.

Theorem 5.3. Let $\mathcal{C}$ denote the complexity function of $u_{\varepsilon, \eta}[c, c+d)$.

- If $d \notin \mathbb{Z}[\varepsilon]$, then

$$
\mathcal{C}(n)=2 n+1, \quad \text { for } n \in \mathbb{N} .
$$

- If $d \in \mathbb{Z}[\varepsilon]$, then there exists a unique non-negative $k \in \mathbb{N}_{0}$ such that $f^{(k)}(\alpha)=\beta$ or $f^{(k+1)}(\beta)=\alpha$, where $\alpha, \beta$ are the discontinuity points of the stepping function $f$. Consequently,

$$
\mathcal{C}(n)=\left\{\begin{array}{cc}
2 n+1 & \text { for } n \leq k \\
n+k+1 & \text { for } n>k
\end{array}\right.
$$


Proof. If $f_{\varepsilon, \Omega}$ and $f_{\varepsilon, \Omega+t}$ are stepping functions corresponding to acceptance intervals $\Omega$ and $\Omega+t$ respectively, then $f_{\varepsilon, \Omega+t}(y)=f_{\varepsilon, \Omega}(y-t)+t$ for any translation $t$ of the interval $\Omega$. This means that blocs occurring in the word $u_{\varepsilon, \eta}[c, c+d)$ occur also in $u_{\varepsilon, \eta}[c+t, c+d+t)$ and vice versa. Two words with such property are said to belong to the same local isomorphism class. The words $u_{\varepsilon, \eta}[c, c+d)$ and $u_{\varepsilon, \eta}[c+t, c+d+t)$ must have the same complexity. Without loss of generality we can thus consider $\Omega=[0, d)$. Recall that $\Delta_{1}^{*}, \Delta_{2}^{*} \in \mathbb{Z}[\varepsilon]$, and that the discontinuity points of $f$ for such an interval $\Omega$ are $\alpha=d-\Delta_{1}^{*}$ and $\beta=-\Delta_{2}^{*}$. Our aim is to determine the number of elements in the set

$$
M=\left\{0, \alpha, f^{(-1)}(\alpha), \ldots, f^{(-(n-1))}(\alpha), \beta, f^{(-1)}(\beta), \ldots, f^{(-(n-1))}(\beta)\right\} .
$$

The function $f^{(k)}$ has no fixed point for any $k$, which implies the following facts:

(i) The points $\alpha, f^{(-1)}(\alpha), \ldots, f^{(-(n-1))}(\alpha)$ are mutually distinct. The same holds if we replace $\alpha$ by $\beta$.

(ii) One can never have simultaneously $f^{k}(\alpha)=\beta$ and $f^{i}(\beta)=\alpha$ for some $k, i \in \mathbb{N}$.

(iii) Since $f(\beta)=0=c$, the point 0 is the closest right neighbour of $\beta^{-*}$ and we have $f^{-i}(\beta) \neq c$ for all $i \in \mathbb{N}_{0}$.

- Suppose that $d \notin \mathbb{Z}[\varepsilon]$. Then $\alpha=d-\Delta_{1}^{*} \notin \mathbb{Z}[\varepsilon]$ and $\beta=-\Delta_{2}^{*} \in$ $\mathbb{Z}[\varepsilon]$. Since $f(y) \in \mathbb{Z}[\varepsilon]$ if and only if $y \in \mathbb{Z}[\varepsilon]$, we have $f^{(-k)}(\alpha) \notin$ $\left\{0, \beta, f^{(-1)}(\beta), \ldots, f^{(-(n-1))}(\beta)\right\}$. Together with properties (i) and (iii) this means that the set $M$ has $2 n+1$ elements.

- Let now $d \in \mathbb{Z}[\varepsilon]$, i.e., the star map images $\alpha^{-*}, \beta^{-*}$ of both discontinuity points of $f$ and the point 0 belong to $\Sigma_{\varepsilon, \eta}(\Omega)$. Since the point 0 is the closest right neighbour of $\beta^{-*}$ we have either $\alpha^{-*} \leq \beta^{-*}$ or $0<\alpha^{-*}$. In the first case it means that there exists a non-negative $k$ such that $\alpha^{-*}$ is the $k$-th left neighbour of $\beta^{-*}$, i.e., $f^{(-i)}(\beta) \neq \alpha$ for $i<k$ and $f^{(-k)}(\beta)=\alpha$. In the second case that there exists a non-negative $k$ such that 0 is the $k$-th left neighbour of $\alpha^{-*}$, i.e., $f^{(-i)}(\alpha) \notin\{0, \beta\}$ for $i<k$ and $f^{(-k)}(\alpha)=0=f(\beta)$. Starting from th value $n-1=k$ the pairs of elements in the set coincide, which influences the cardinality of this set and consequently also the complexity of the infinite word.

Since infinite words with complexity $n+$ const. (quasisturmian sequences, see [8]) are well described, we shall now focus on words of complexity $2 n+1$. According to the result of Alessandri and Berthé [2,7], for a given $n \in \mathbb{N}$ the densities of factors of length $n$ can take at most $3(\mathcal{C}(n+1)-\mathcal{C}(n))=6$ values. In fact, there are only five frequencies for factors of given length. 
This can be proved following the ideas in [2] and using the fact that the words associated to cut-and-project sequences are codings of three interval exchange.

Let us determine the maximal in- and outdegree in the Rauzy graphs corresponding to the infinite words in consideration. Let $e$ be a factor of length $n+1$ in the infinite word $u_{\varepsilon, \eta}[c, c+d)$ and let $v$ be the prefix of $e$ of length $n$. Obviously $\Omega_{e} \subseteq \Omega_{v}$. Therefore in the Rauzy graph $\Gamma_{n}$ the vertex $v$ is the starting point of the edge $e$. If $\Omega_{e}=\Omega_{v}$, then the outdegree of the vertex $v$ is equal to one. This happens only if the points $f^{(-n)}(\alpha), f^{(-n)}(\beta)$ do not belong to the interval $\Omega_{v}$. If the interval $\Omega_{v}$ contains exactly one of the points $f^{(-n)}(\alpha), f^{(-n)}(\beta)$, then $\Omega_{v}=\Omega_{e_{1}} \cup \Omega_{e_{2}}$, where $e_{1}, e_{2}$ are the only two factors of length $n+1$ with prefix $v$ and the outdegree of the vertex $v$ is equal to 2 . In case that both points $f^{(-n)}(\alpha)$, $f^{(-n)}(\beta)$ happen to belong to the interval $\Omega_{v}$, the outdegree of the vertex $v$ is equal to 3 and all other vertices in the Rauzy graph $\Gamma_{n}$ have outdegree 1 . Since the set $\left\{f^{(-n)}(\alpha), f^{(-n)}(\beta) \mid n \in N\right\}$ covers the acceptance interval $\Omega$ densely and uniformly [22], the lengths of intervals $\Omega_{v}$ tend to 0 as $n$ tends to infinity. If for every $n \in \mathbb{N}$ both of the points $f^{(-n)}(\alpha)$ and $f^{(-n)}(\beta)$ fall into the same interval $\Omega_{v}$ for some factor $v$ of length $n$, then $\lim _{n \rightarrow \infty}\left(f^{(-n)}(\alpha)-f^{(-n)}(\beta)\right)=0$. Take $n$ large enough so that $\mid f^{(-n)}(\alpha)-$ $f^{(-n)}(\beta) \mid<\delta$. From the properties of the stepping function, as piecewise linear function with slope 1 , it can happen that $\left|f^{(-n-1)}(\alpha)-f^{(-n-1)}(\beta)\right|=$ $\left|f^{(-n)}(\alpha)-f^{(-n)}(\beta)\right|$. However, this cannot be true for every $n$. In the opposite case, we have $\left|f^{(-n-1)}(\alpha)-f^{(-n-1)}(\beta)\right| \gg\left|f^{(-n)}(\alpha)-f^{(-n)}(\beta)\right|$ and thus points $f^{(-n-1)}(\alpha), f^{(-n-1)}(\beta)$ cannot be in the same interval $\Omega_{v}$. Thus we have proved the following statement.

Proposition 5.4. Let $\varepsilon, \eta$ be irrational numbers, $\varepsilon \neq-\eta$, and let $\Omega=$ $[c, c+d)$ be a non empty interval. Then there exists an $n_{0}$ such that for all $n \geq n_{0}$ the maximal indegree and the maximal outdegree in the Rauzy graph $\Gamma_{n}$ of $u_{\varepsilon, \eta}(\Omega)$ are equal to 2.

\section{Geometrically similar cut-and-project sequences}

Two sets $\Lambda$ and $\hat{\Lambda} \subset \mathbb{R}$ are geometrically similar, if there exist $\varphi, \psi \in \mathbb{R}$, $\varphi \neq 0$, such that $\hat{\Lambda}=\phi \Lambda+\psi$. We shall denote this property by $\Lambda \stackrel{\mathbb{g}}{\sim} \hat{\Lambda}$.

Remark 6.1. Note that if two cut-and-project sequences $\Sigma_{\varepsilon, \eta}(\Omega)$ and $\Sigma_{\hat{\varepsilon}, \hat{\eta}}(\hat{\Omega})$ are geometrically similar with a positive similarity factor $\varphi$, then the corresponding infinite bidirectional words $u_{\varepsilon, \eta}(\Omega)$ and $u_{\hat{\varepsilon}, \hat{\eta}}(\hat{\Omega})$ coincide. A converse statement is not true, cf. Proposition 4.4.

In order to describe classes of mutually geometrically similar cut-andproject sequences, let us consider the group of symmetries of the lattice $\mathbb{Z}^{2}$. 
We have the translations $\mathbb{Z}^{2}+\left(\begin{array}{l}a \\ b\end{array}\right)=\mathbb{Z}^{2}$, for $a, b \in \mathbb{Z}$, and the group of rotation symmetries of $\mathbb{Z}^{2}$. They are given by all integer valued matrices $\mathbb{A}$ with determinant \pm 1 . Then $\mathbb{A Z}^{2}=\mathbb{Z}^{2}$. These symmetries of $\mathbb{Z}^{2}$ correspond to the following transformations of cut-and-project sequences.

Proposition 6.2. Let $\varepsilon, \eta$ be irrational numbers, $\varepsilon \neq-\eta$, and let $\Omega$ be a bounded interval.

- For $x \in \mathbb{Z}[\eta]$ we have

$$
\Sigma_{\varepsilon, \eta}(\Omega)+x=\Sigma_{\varepsilon, \eta}\left(\Omega+x^{*}\right)
$$

- Let $a, b, c, d$ be integers, such that $a d-b c= \pm 1$. Then

$$
\Sigma_{\varepsilon, \eta}(\Omega)=(a+c \eta) \Sigma_{\frac{-b+d \varepsilon}{a-c \varepsilon}, \frac{b+d \eta}{a+c \eta}}\left(\frac{1}{a-c \varepsilon} \Omega\right) .
$$

Proof. First, let $x=a+b \eta \in \mathbb{Z}[\eta]$. Then

$$
\begin{aligned}
\Sigma_{\varepsilon, \eta}(\Omega)+x & =\{(p+a)+(q+b) \eta \mid p, q \in \mathbb{Z}, p-q \varepsilon \in \Omega\} \\
& =\{p+q \eta \mid p, q \in \mathbb{Z},(p-a)-(q-b) \varepsilon \in \Omega\} \\
& =\{p+q \eta \mid p, q \in \mathbb{Z}, p-q \varepsilon \in \Omega+a-b \varepsilon\} \\
& =\Sigma_{\varepsilon, \eta}\left(\Omega+x^{*}\right)
\end{aligned}
$$

Now let $\mathbb{A}=\left(\begin{array}{ll}a & b \\ c & d\end{array}\right)$ be an integer valued matrix with determinant \pm 1 . Then $\mathbb{A Z}^{2}=\mathbb{Z}^{2}$ and therefore

$$
\begin{aligned}
\Sigma_{\varepsilon, \eta}(\Omega) & =\{p+q \eta \mid p, q \in \mathbb{Z}, p-q \varepsilon \in \Omega\} \\
& =\left\{(1, \eta)\left(\begin{array}{l}
p \\
q
\end{array}\right) \mid p, q \in \mathbb{Z},(1,-\varepsilon)\left(\begin{array}{l}
p \\
q
\end{array}\right) \in \Omega\right\} \\
& =\left\{(1, \eta) \mathbb{A}\left(\begin{array}{c}
p \\
q
\end{array}\right) \mid p, q \in \mathbb{Z},(1,-\varepsilon) \mathbb{A}\left(\begin{array}{l}
p \\
q
\end{array}\right) \in \Omega\right\} \\
& =\left\{(a+c \eta, b+d \eta)\left(\begin{array}{l}
p \\
q
\end{array}\right) \mid p, q \in \mathbb{Z},(a-c \varepsilon, b-d \varepsilon)\left(\begin{array}{l}
p \\
q
\end{array}\right) \in \Omega\right\} \\
& =(a+c \eta)\left\{\left(1, \frac{b+d \eta}{a+c \eta}\right)\left(\begin{array}{l}
p \\
q
\end{array}\right) \mid p, q \in \mathbb{Z},\left(1, \frac{b-d \varepsilon}{a-c \varepsilon}\right)\left(\begin{array}{l}
p \\
q
\end{array}\right) \in \frac{1}{a-c \varepsilon} \Omega\right\} \\
& =(a+c \eta) \Sigma_{\frac{-b+d \varepsilon}{a-c \varepsilon}, \frac{b+d \eta}{a+c \eta}}\left(\frac{1}{a-c \varepsilon} \Omega\right) .
\end{aligned}
$$


The group of integer valued matrices with determinant \pm 1 is generated by matrices $\mathbb{A}_{1}=\left(\begin{array}{ll}1 & 1 \\ 0 & 1\end{array}\right), \mathbb{A}_{2}=\left(\begin{array}{cc}1 & 0 \\ 0 & -1\end{array}\right), \mathbb{A}_{3}=\left(\begin{array}{ll}0 & 1 \\ 1 & 0\end{array}\right)$. Elementary transformations of cut-and-project sequences for these matrices are

$$
\begin{aligned}
& \Sigma_{\varepsilon, \eta}(\Omega)=\Sigma_{\varepsilon-1, \eta+1}(\Omega), \\
& \Sigma_{\varepsilon, \eta}(\Omega)=\Sigma_{-\varepsilon,-\eta}(-\Omega), \\
& \Sigma_{\varepsilon, \eta}(\Omega)=\eta \Sigma_{\frac{1}{\varepsilon}, \frac{1}{\eta}}\left(-\frac{1}{\varepsilon} \Omega\right) .
\end{aligned}
$$

Using the above elementary transformation we show that we may limit our considerations to $\varepsilon, \eta$ and $\Omega$ with certain properties, without loosing any infinite bidirectional word. The aim of this section is to prove the following theorem.

Theorem 6.3. Let $\varepsilon, \eta$ are irrational numbers, $\varepsilon \neq-\eta$. Let $\Omega$ be a bounded interval. Then there exist irrational numbers $\hat{\varepsilon}, \hat{\eta}$ and an interval $\hat{\Omega}$, such that

$$
\Sigma_{\varepsilon, \eta}(\Omega) \stackrel{\mathbb{g}}{\sim} \Sigma_{\hat{\varepsilon}, \hat{\eta}}(\hat{\Omega}), \quad 0<\hat{\varepsilon}<1, \hat{\eta}>0, \quad \max (1-\hat{\varepsilon}, \hat{\varepsilon})<|\hat{\Omega}| \leq 1 .
$$

The proof of the theorem will be divided into two lemmas. Before stating the lemmas, let us recall certain properties of continued fractions [12].

If $\xi>0$ is an irrational number with continued fraction $\left[a_{0}, a_{1}, a_{2}, \ldots\right]$ and $\left(\frac{p_{n}}{q_{n}}\right)_{n \in \mathbb{N}_{0}}$ the sequence of the convergents associated to $\xi$, then for every $n \in \mathbb{N}_{0}$ we have

$$
\begin{gathered}
\frac{p_{2 n}}{q_{2 n}}<\xi<\frac{p_{2 n+1}}{q_{2 n+1}}, \\
\frac{1}{q_{n+2}}<\left|q_{n} \xi-p_{n}\right|<\frac{1}{q_{n+1}}, \\
p_{n+1} q_{n}-p_{n} q_{n+1}=(-1)^{n} .
\end{gathered}
$$

Lemma 6.4. For any irrational $\varepsilon, \eta, \varepsilon \neq-\eta$, and bounded interval $\Omega$, there exist irrational numbers $\hat{\varepsilon}, \hat{\eta}$ and a bounded interval $\hat{\Omega}$ such that

$$
\Sigma_{\varepsilon, \eta}(\Omega) \stackrel{g}{\sim} \Sigma_{\hat{\varepsilon}, \hat{\eta}}(\hat{\Omega}), \quad \hat{\varepsilon}>0, \hat{\eta}>0, \quad \text { and } \quad|\hat{\Omega}| \leq 1 .
$$

Proof. Using transformation (12) we may assume without loss of generality that $\eta>0$. We first find convergents $\frac{p_{2 n}}{q_{2 n}}$ and $\frac{p_{2 n+1}}{q_{2 n+1}}$ associated to $\eta$, so that $-\varepsilon \notin\left[\frac{p_{2 n}}{q_{2 n}}, \frac{p_{2 n+1}}{q_{2 n+1}}\right]$. From the property (i) of continued fractions we have

$$
\frac{p_{2 n}}{q_{2 n}}<\eta<\frac{p_{2 n+1}}{q_{2 n+1}} \Longleftrightarrow \frac{-p_{2 n}+\eta q_{2 n}}{p_{2 n+1}-\eta q_{2 n+1}}>0
$$

and

$$
\left(\frac{p_{2 n}}{q_{2 n}}+\varepsilon\right)\left(\frac{p_{2 n+1}}{q_{2 n+1}}+\varepsilon\right)>0 \Longleftrightarrow \frac{p_{2 n}+\varepsilon q_{2 n}}{p_{2 n+1}+\varepsilon q_{2 n+1}}>0
$$


Let us define the matrix $\mathbb{A}=\left(\begin{array}{ll}a & b \\ c & d\end{array}\right)=\left(\begin{array}{cc}p_{2 n+1} & -p_{2 n} \\ -q_{2 n+1} & q_{2 n}\end{array}\right)$ for transformation (10). Then from (14) we have

$$
\hat{\eta}:=\frac{b+d \eta}{a+c \eta}=\frac{-p_{2 n}+\eta q_{2 n}}{p_{2 n+1}-\eta q_{2 n+1}}>0
$$

and from (15) we have

$$
\hat{\varepsilon}:=\frac{-b+d \varepsilon}{a-c \varepsilon}=\frac{p_{2 n}+\varepsilon q_{2 n}}{p_{2 n+1}+\varepsilon q_{2 n+1}}>0 .
$$

From the property (iii) of continued fractions we obtain

$$
\operatorname{det} \mathbb{A}=p_{2 n+1} q_{2 n}-p_{2 n} q_{2 n+1}=1 \text {. }
$$

In the transformation (10) with the matrix $\mathbb{A}$ we obtain a new acceptance window $\hat{\Omega}:=\frac{1}{a-c \varepsilon} \Omega$. Let us find an estimate of the denominator in the fraction

$$
\begin{aligned}
|a-c \varepsilon|=|c|\left|\eta+\varepsilon-\left(\frac{a}{c}+\eta\right)\right| & \geq q_{2 n+1}|\eta+\varepsilon|-\left|q_{2 n+1} \eta-p_{2 n+1}\right| \\
& \geq q_{2 n+1}|\eta+\varepsilon|-\frac{1}{q_{2 n+2}} \\
& \geq q_{2 n+1}|\eta+\varepsilon|-1 .
\end{aligned}
$$

Note that in the estimate we have used property (ii) of continued fractions. Since $\lim _{n \rightarrow \infty} q_{2 n+1}=+\infty$ and $|\eta+\varepsilon| \neq 0$, it is possible to choose a sufficiently large $n$, so that the length of the interval $\hat{\Omega}$ is smaller or equal to 1 .

Lemma 6.5. Let $\varepsilon, \eta>0, \varepsilon, \eta$ irrational and let $\Omega$ be an interval of length $|\Omega| \leq 1$. Then there exist irrational numbers $\hat{\varepsilon}, \hat{\eta}$ and an interval $\hat{\Omega}$ satisfying

$$
\Sigma_{\varepsilon, \eta}(\Omega) \stackrel{\mathbb{g}}{\sim} \Sigma_{\hat{\varepsilon}, \hat{\eta}}(\hat{\Omega}), \quad 0<\hat{\varepsilon}<1, \hat{\eta}>0, \quad \max (1-\hat{\varepsilon}, \hat{\varepsilon})<|\hat{\Omega}| \leq 1 .
$$

Proof. Without loss of generality we may assume that $\varepsilon<1$. Otherwise we would use the transformation (11) to get

$$
\Sigma_{\varepsilon, \eta}(\Omega)=\Sigma_{\varepsilon-[\varepsilon], \eta+[\varepsilon]}(\Omega),
$$

where $\varepsilon-[\varepsilon]<1$. We first show that there exist $0<\check{\varepsilon}<1, \check{\eta}>0$ and $\check{\Omega}$ such that

$$
\Sigma_{\varepsilon, \eta}(\Omega) \stackrel{\stackrel{\Omega}{\sim}}{\sim} \Sigma_{\check{\varepsilon}, \check{\eta}}(\check{\Omega}), \quad \text { and } \quad \check{\varepsilon}<|\check{\Omega}| \leq 1 .
$$

The case of $|\Omega|>\varepsilon$ is trivial, because it suffices to put $\check{\varepsilon}=\varepsilon, \check{\eta}=\eta$ and $\check{\Omega}=\Omega$. Assume that for given $\Omega$ we have $|\Omega| \leq \varepsilon$. Let $\left(\frac{p_{n}}{q_{n}}\right)_{n \in \mathbb{N}_{0}}$ be the convergents associated to $\varepsilon$. According to property (ii) of continued fractions, the sequence $\left(\left|p_{n}-q_{n} \varepsilon\right|\right)_{n \in \mathbb{N}_{0}}$ is decreasing and thus it is possible to find $n \in \mathbb{N}$ so that

$$
\left|p_{n}-q_{n} \varepsilon\right|<|\Omega| \leq\left|p_{n-1}-q_{n-1} \varepsilon\right| \leq\left|p_{0}-q_{0} \varepsilon\right|=\varepsilon .
$$


Let us transform the cut-and-project set $\Sigma_{\varepsilon, \eta}(\Omega)$ using the transforma-

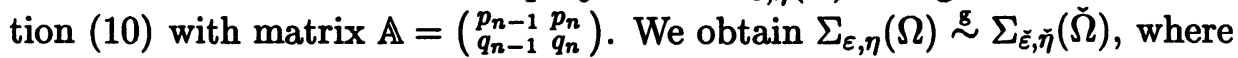

$$
\check{\varepsilon}=\frac{-p_{n}+q_{n} \varepsilon}{p_{n-1}-q_{n-1} \varepsilon}, \quad \check{\eta}=\frac{p_{n}+q_{n} \eta}{p_{n-1}+q_{n-1} \eta}, \quad \check{\Omega}=\frac{1}{p_{n-1}-q_{n-1} \varepsilon} \Omega .
$$

According to properties (i) and (ii) of the continued fractions we have $0<\check{\varepsilon}<1$. The number $\check{\eta}$ is clearly positive. Since for $|\Omega|$ we have (16), for the length of $\check{\Omega}$ we may write

$$
\check{\varepsilon}=\frac{-p_{n}+q_{n} \varepsilon}{p_{n-1}-q_{n-1} \varepsilon}<|\check{\Omega}| \leq 1,
$$

what was to be shown.

If moreover $|\check{\Omega}|>1-\check{\varepsilon}$, we may set $\hat{\varepsilon}:=\check{\varepsilon}, \hat{\eta}:=\check{\eta}$, and $\hat{\Omega}:=\check{\Omega}$, and the lemma is proved. It thus remains to solve the case

$$
\check{\varepsilon}<|\check{\Omega}| \leq 1-\check{\varepsilon} \text {. }
$$

This, however, is possible only if $\check{\varepsilon}<\frac{1}{2}$, i.e., its continued fraction has the form $\check{\varepsilon}=\left[0, c_{1}, c_{2}, \ldots\right]$ with $c_{1} \geq 2$. Since

$$
1-\check{\varepsilon}>1-2 \check{\varepsilon}>\cdots>1-\left(c_{1}-1\right) \check{\varepsilon}>\check{\varepsilon},
$$

it is possible to find a minimal $s \in\left\{1,2, \ldots, c_{1}-1\right\}$ so that

$$
|\check{\Omega}| \leq 1-s \check{\varepsilon} \text {. }
$$

Now we use the transformation (10) with the matrix $\mathbb{A}=\left(\begin{array}{ll}1 & 0 \\ s & 1\end{array}\right)$ to get $\Sigma_{\hat{\varepsilon}, \hat{\eta}}(\hat{\Omega}) \stackrel{g}{\sim} \Sigma_{\check{\varepsilon}, \grave{\eta}}(\check{\Omega})$, for

$$
\hat{\varepsilon}:=\frac{\check{\varepsilon}}{1-s \check{\varepsilon}}, \quad \hat{\eta}:=\frac{\check{\eta}}{1+s \check{\eta}}, \quad \hat{\Omega}:=\frac{1}{1-s \check{\varepsilon}} \check{\Omega} .
$$

Let us verify that $\hat{\varepsilon}, \hat{\eta}$ and $\hat{\Omega}$ satisfy the inequalities required by the lemma. The parameter $\hat{\eta}$ is positive as it is a ratio of positive numbers. For $\hat{\varepsilon}$ we use the inequality (18) to obtain $0<\hat{\varepsilon}<1$. For the estimate of $\hat{\Omega}$ we derive from (17) and (19) that

$$
\hat{\varepsilon}=\frac{\check{\varepsilon}}{1-s \check{\varepsilon}}<|\hat{\Omega}| \leq 1 .
$$

In order to complete the proof of the lemma, it remains to show that $|\hat{\Omega}|>1-\hat{\varepsilon}$. If the minimal $s$ satisfies $s \leq c_{1}-2$, we have $1-(s+1) \check{\varepsilon}<$ $|\check{\Omega}| \leq 1-s \check{\varepsilon}$ which implies

$$
1 \geq|\hat{\Omega}|>\frac{1-(s+1) \check{\varepsilon}}{1-s \check{\varepsilon}}=1-\frac{\check{\varepsilon}}{1-s \check{\varepsilon}}=1-\hat{\varepsilon} .
$$

If the minimal $s$ is $s=c_{1}-1$, then automatically $\hat{\varepsilon}>1-\hat{\varepsilon}$ which, together with (20) implies in both cases $1 \geq|\hat{\Omega}|>\max (\hat{\varepsilon}, 1-\hat{\varepsilon})$ and proves the lemma.

Combination of Lemmas 6.4 and 6.5 constitutes the proof of Theorem 6.3. 
Remark 6.6. Note that in Theorem 6.3 we may choose the interval $\hat{\Omega}$ in such a way that the similarity factor between $\Sigma_{\varepsilon, \eta}(\Omega)$ and $\Sigma_{\hat{\varepsilon}, \hat{\eta}}(\hat{\Omega})$ is positive, thus infinite bidirectional words associated to these cut-and-project sets are identical. If it happens that $\Sigma_{\varepsilon, \eta}(\Omega)=\varphi \Sigma_{\hat{\varepsilon}, \hat{\eta}}(\hat{\Omega})$ for some $\varphi<0$, we can use the transformation matrix $\mathbb{A}=\left(\begin{array}{cc}-1 & 0 \\ 0 & -1\end{array}\right)$ to obtain

$$
\Sigma_{\hat{\varepsilon}, \hat{\eta}}(\hat{\Omega})=-\Sigma_{\hat{\varepsilon}, \hat{\eta}}(-\hat{\Omega}) .
$$

Therefore using the interval $-\hat{\Omega}$ instead of $\hat{\Omega}$ gives us a positive similarity factor $-\varphi$.

\section{Combinatorial classification of cut-and-project sequences}

Let us recall that two words $u=\left(u_{n}\right)_{n \in \mathbb{Z}}$ and $\hat{u}=\left(\hat{u}_{n}\right)_{n \in \mathbb{Z}}$ in alphabets $\mathcal{A}$ and $\hat{\mathcal{A}}$, respectively, are combinatorially equivalent, if there exists a bijection $h: \mathcal{A} \rightarrow \hat{\mathcal{A}}$ and an $n_{0} \in \mathbb{Z}$ such that $\hat{u}_{n}=h\left(u_{n+n_{0}}\right)$ for all $n \in \mathbb{Z}$. We denote this by $u \stackrel{c}{\sim} \hat{u}$. Note that if a bijection $h$ should exist, certainly the density of a letter $a$ in the word $u$ must be the same as the density of $h(a)$ in $\hat{u}$.

Remark 7.1. Recall that two words associated to cut-and-project sets which are geometrically similar with positive similarity factor are combinatorially equivalent, see Remark 6.1. Using Theorem 6.3 and Remark 6.6 we may thus limit our consideration, without loss of generality, to words $u_{\varepsilon, \eta}(\Omega)$ with parameters satisfying

$$
0<\varepsilon<1, \quad \eta>0, \quad \max (\varepsilon, 1-\varepsilon)<|\Omega| \leq 1 .
$$

In this case the stepping function has the form :

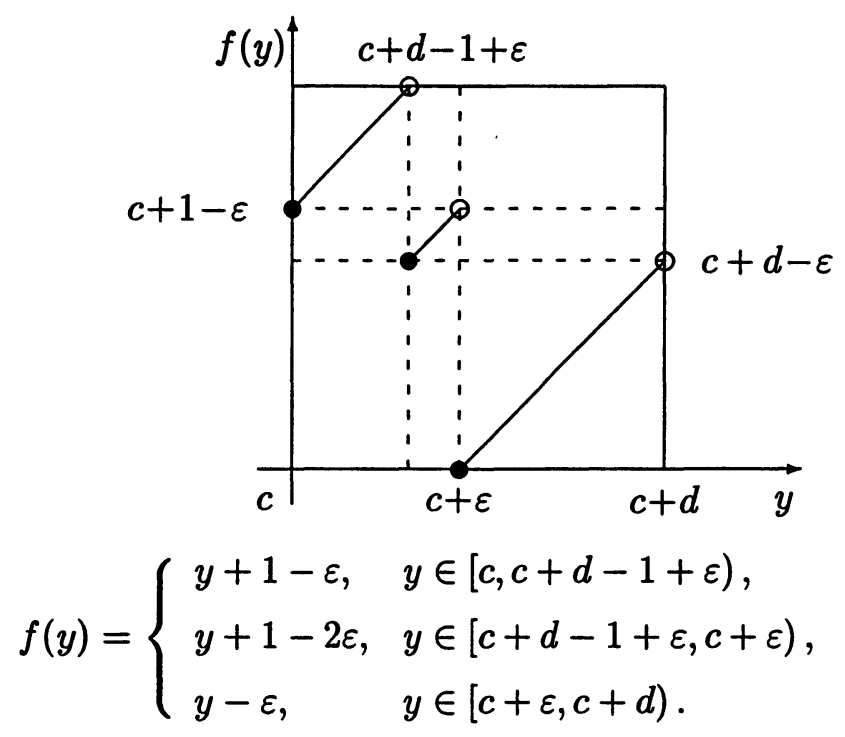


and thus we have

$\Omega_{C}=[c+\varepsilon, c+d), \quad \Omega_{B}=[c, c+d-1+\varepsilon), \quad \Omega_{A}=[c+d-1+\varepsilon, c+\varepsilon)$.

For the densities of tiles $A, B$, and $C$ we have in this case

$$
\varrho_{A}=\frac{1}{d}-1, \quad \varrho_{B}=1-\frac{1-\varepsilon}{d}, \quad \text { and } \quad \varrho_{C}=1-\frac{\varepsilon}{d},
$$

respectively, cf. Proposition 5.2.

Proposition 4.4 shows that two geometrically non-similar cut-and-project sequences may correspond to combinatorially equivalent infinite words. Let us show yet another example of combinatorially equivalent words.

Example 2. Consider Sturmian words $u, \hat{u}$ in the alphabet $\{0,1\}$ with the slope $\alpha$, and $\hat{\alpha}=1-\alpha$ respectively, where $0<\alpha<1$. Obviously, $u$ and $\hat{u}$ are combinatorially equivalent since $\hat{u}$ arises from $u$ by replacing 0 's with 1 's and vice versa. Such Sturmian words correspond to cut-and-project sequences with $\Omega$ a semi-closed interval of length 1, see Example 1. For $\eta>0$ we have

$$
\begin{aligned}
\Sigma_{\alpha, \eta}[c, c+1) & =\left\{x_{n} \equiv\lceil c+n \alpha\rceil+n \eta \mid n \in \mathbb{Z}\right\} \\
\Sigma_{1-\alpha, \eta}(-c-1,-c] & =\left\{y_{n} \equiv\lfloor-c+(1-\alpha) n\rfloor+n \eta \mid n \in \mathbb{Z}\right\} .
\end{aligned}
$$

Both of the sets have two tiles of lengths $\eta$ and $\eta+1$. More precisely, the distances are

$$
\begin{aligned}
& x_{n+1}-x_{n}=\eta+\lceil c+n \alpha+\alpha\rceil-\lceil c+n \alpha\rceil, \\
& y_{n+1}-y_{n}=\eta+1-\lceil c+n \alpha+\alpha\rceil+\lceil c+n \alpha\rceil .
\end{aligned}
$$

We can see that

$$
\begin{aligned}
& x_{n+1}-x_{n}=\eta \\
& x_{n+1}-x_{n}=\eta+1 \quad \Longrightarrow \quad y_{n+1}-y_{n}=\eta+1 \\
& y_{n+1}-y_{n}=\eta
\end{aligned}
$$

The above example illustrates that for acceptance window of length 1 the interchange $\varepsilon \leftrightarrow 1-\varepsilon, \Omega \leftrightarrow-\Omega$ gives combinatorially equivalent words. The following lemma states that the same property holds for general acceptance window as well.

Lemma 7.2. Let $0<\varepsilon<1, \eta>0$ be irrational numbers, and let $\Omega$ be an interval of length $\max (\varepsilon, 1-\varepsilon)<|\Omega| \leq 1$. Then

$$
u_{\varepsilon, \eta}(-\Omega) \stackrel{\mathrm{c}}{\sim} u_{1-\varepsilon, \eta}(\Omega) \text {. }
$$

Proof. Although $\mathbb{Z}[\varepsilon]=\mathbb{Z}[1-\varepsilon]$, the star maps from $\mathbb{Z}[\eta]$ to $\mathbb{Z}[\varepsilon]=\mathbb{Z}[1-\varepsilon]$ are different for the cut-and-project sequences $\Sigma_{\varepsilon, \eta}(-\Omega)$ and $\Sigma_{1-\varepsilon, \eta}(\Omega)$. We shall therefore distinguish them by indices,

$$
\begin{array}{lll}
*_{1}: p+q \eta \mapsto p-q \varepsilon & \text { for } \Sigma_{\varepsilon, \eta}(-\Omega), \\
*_{2}: p+q \eta \mapsto p-q(1-\varepsilon) & \text { for } \Sigma_{1-\varepsilon, \eta}(\Omega) .
\end{array}
$$


According to Proposition 4.3 in both cut-and-project sets the three distances are $\eta, 1+\eta$ and $1+2 \eta$. Their images under the star map are however different for $*_{1}$ and $*_{2}$, namely,

$$
\begin{array}{rrrr}
-\varepsilon, & 1-\varepsilon, & 1-2 \varepsilon, & \text { for } *_{1}, \\
\varepsilon-1, & \varepsilon, & 2 \varepsilon-1, & \text { for } *_{2}
\end{array}
$$

For the proof we use two facts:

(F1) $f_{\varepsilon, \Omega}^{-1}(x)=f_{1-\varepsilon, \Omega}(x)$ for all $x \in \Omega$,

(F2) $\quad \Sigma_{\varepsilon, \eta}(\Omega)=-\Sigma_{\varepsilon, \eta}(-\Omega)$.

Let $x_{0} \in \Omega \cap \mathbb{Z}[\varepsilon]$. Then the fact (F2) says that the sequence of distances to the left from the point $x_{0}^{-*_{1}}$ in the set $\Sigma_{\varepsilon, \eta}(\Omega)$ is the same as the sequence of distances to the right from the point $-x_{0}^{-*_{1}}$ in the set $\Sigma_{\varepsilon, \eta}(-\Omega)$.

The fact (F1) says that the sequence of steps to the right from the point $x_{0}^{-*_{2}}$ in the set $\Sigma_{1-\varepsilon, \eta}(\Omega)$ is the same as the sequence of steps to the left from the point $x_{0}^{-*_{1}}$ in the set $\Sigma_{\varepsilon, \eta}(\Omega)$, only the length of steps $\eta$ and $1+\eta$ are interchanged.

Note that the density of the shortest tile $\eta$ in the set $\Sigma_{\varepsilon, \eta}(-\Omega)$ is equal to $1-\frac{\varepsilon}{d}$, whereas the density of the shortest tile $\eta$ in the set $\Sigma_{1-\varepsilon, \eta}(\Omega)$ is equal to $1-\frac{1-\varepsilon}{d}$. It is therefore obvious that the cut-and-project sets $\Sigma_{\varepsilon, \eta}(-\Omega)$ and $\Sigma_{1-\varepsilon, \eta}(\Omega)$ are not geometrically similar. Nevertheless, the associated infinite bidirectional words are combinatorially equivalent.

From every Sturmian sequence in the alphabet $\{A, B\}$ we can form a special word in the alphabet $\{A, B, C\}$ in the way that in between every two letters of the Sturmian sequence we insert $k$-times the letter $C$. Such a sequence we call a $k$-padded Sturmian sequence.

Definition 7.3. Let $u$ be a Sturmian word in the alphabet $\{A, B\}$. Let $k \in \mathbb{N}$ and $h$ be a morphism $h:\{A, B\} \rightarrow\{A, B, C\}^{*}$, given by $h(A)=A C^{k}$, $h(B)=B C^{k}$. The word $h(u)$ is called a $k$-padded Sturmian word.

The following proposition shows that $k$-padded Sturmian sequences can also be cut-and-project sequences.

Proposition 7.4. Every $k$-padded Sturmian word is a word associated to a cut-and-project sequence.

Proof. Let $u$ be a Sturmian word with slope $\alpha, 0<\alpha<1$. Similarly as in Examples 1 and 2 we can consider $u$ to be the word $u_{\alpha, \mu}[c, c+1)$, for some $c \in \mathbb{R}$ and $\mu>0$. For a given $k \in \mathbb{N}$ we form a $k$-padded Sturmian sequence and want to find parameters $\varepsilon, \eta, \Omega$, so that the $k$-padded Sturmian word is a word $u_{\varepsilon, \eta}(\Omega)$. We put

$$
\varepsilon=\frac{1}{k+2-\alpha}, \quad \eta=\mu, \quad \Omega=[\varepsilon c, \varepsilon(c+k+1)) .
$$


Note that $\Omega$ can be written as $\Omega=[\tilde{c}, \tilde{c}+\tilde{d})$ for $\tilde{c}=\varepsilon c$ and $\tilde{d}=(k+1) \varepsilon$. Since $\frac{1}{k+2}<\varepsilon<\frac{1}{k+1}$, we have $\max \{1-\varepsilon, \varepsilon\}<\tilde{d}<1$ and therefore star images of tiles in $\Sigma_{\varepsilon, \eta}(\Omega)$ are $\varepsilon, 1-\varepsilon$, and $1-2 \varepsilon$.

For such parameters the stepping function $f_{\varepsilon, \Omega}$ is given by

$$
f_{\varepsilon, \Omega}(y):=\left\{\begin{array}{lll}
y+1-\varepsilon & \text { for } y \in[\varepsilon c,(c+k+2) \varepsilon-1) & =\Omega_{A}, \\
y+1-2 \varepsilon & \text { for } y \in[(c+k+2) \varepsilon-1, \varepsilon c+\varepsilon) & =\Omega_{B}, \\
y-\varepsilon & \text { for } y \in[\varepsilon c+\varepsilon,(c+k+1) \varepsilon) & =\Omega_{C} .
\end{array}\right.
$$

We can verify easily that

$$
\begin{aligned}
f_{\varepsilon, \Omega}^{(i)}\left(\Omega_{A} \cup \Omega_{B}\right) & \subseteq \Omega_{C}, \quad \text { for } i=1,2, \ldots, k, \\
f_{\varepsilon, \Omega}^{(k+1)}\left(\Omega_{A} \cup \Omega_{B}\right) & =\left(\Omega_{A} \cup \Omega_{B}\right) .
\end{aligned}
$$

Therefore every letter $A$ or $B$ is followed by the string of $k$ letters $C$, whereas the $(k+1)$-th letter is different from $C$. Moreover, the restriction of $f_{\varepsilon, \Omega}^{(k+1)}$ to $\Omega_{A} \cup \Omega_{B}$ is a scaled stepping function $f_{\alpha,[c, c+1)}$.

\section{Theorem 7.5.}

- For any irrational numbers $\varepsilon, \eta, \varepsilon \neq-\eta$, and a bounded interval $\Omega$ there exist irrational $\hat{\varepsilon}$ and an interval $\hat{\Omega}$ satisfying

$$
0<\hat{\varepsilon}<\frac{1}{2}, \quad 1-\hat{\varepsilon}<|\hat{\Omega}| \leq 1,
$$

such that $u_{\varepsilon, \eta}(\Omega) \stackrel{\mathcal{c}}{\sim} u_{\hat{\varepsilon}, \hat{\eta}}(\hat{\Omega})$ for any irrational $\hat{\eta}>0$.

- Let $\varepsilon, \hat{\varepsilon}, \eta, \hat{\eta}$ be positive irrational numbers such that $0<\varepsilon, \hat{\varepsilon}<\frac{1}{2}$. Let $\Omega, \hat{\Omega}$ be intervals satisfying $1-\varepsilon<|\Omega| \leq 1,1-\hat{\varepsilon}<|\hat{\Omega}| \leq 1$, $u_{\varepsilon, \eta}(\Omega) \stackrel{c}{\sim} u_{\hat{\varepsilon}, \hat{\eta}}(\hat{\Omega})$. Then

- either $\varepsilon=\hat{\varepsilon}$ and there exists $x \in \mathbb{Z}[\varepsilon]$ such that $\Omega \cap \mathbb{Z}[\varepsilon]=(x+\hat{\Omega}) \cap$ $\mathbb{Z}[\varepsilon]$, i.e., $u_{\varepsilon, \eta}(\Omega)=u_{\hat{\varepsilon}, \hat{\eta}}(\hat{\Omega})$.

- or there exists $k \in \mathbb{N}$, and an irrational $\alpha, 0<\alpha<1$, such that $u_{\varepsilon, \eta}(\Omega)$ is $k$-padded Sturmian word with slope $\alpha$ and $u_{\hat{\varepsilon}, \hat{\eta}}(\hat{\Omega})$ is a $k$ padded Sturmian word with slope $1-\alpha$.

The proof of the first statement of the theorem is a direct consequence of Lemma 7.2 and Remark 7.1. In the remaining part of the section we are going to prove the second statement.

Let us determine using the stepping function the possible lengths of blocks of one letter in the word $u_{\varepsilon, \eta}(\Omega)$. Let us denote by $\min (A)$, $\max (A)$, (resp. $\min (B), \max (B)$ ) the minimal and the maximal number of letters $C$ that follow a letter $A$ (resp. $B$ ) in the word $u_{\varepsilon, \eta}(\Omega)$. 
Lemma 7.6. Let $0<\varepsilon<\frac{1}{2}, \eta>0$ be irrational numbers, and let $\Omega$ be an interval of length $1-\varepsilon<|\Omega| \leq 1$. Then

$$
\begin{array}{ll}
\min (A)=\left\lfloor d \varepsilon^{-1}\right\rfloor-1, & \max (A)=\left\lfloor\varepsilon^{-1}\right\rfloor-1, \\
\min (B)=\left\lfloor\varepsilon^{-1}\right\rfloor-1, & \max (B)=\left\lceil d \varepsilon^{-1}\right\rceil-1 .
\end{array}
$$

Proof. Let us rewrite formally the definition of $\max (A)$,

We have

$$
\max (A)=\max \left\{k \mid \forall i=1,2, \ldots, k: \Omega_{C} \cap f^{(i)}\left(\Omega_{A}\right) \neq \emptyset\right\} .
$$

$$
f\left(\Omega_{A}\right)=f([c+d-1+\varepsilon, c+\varepsilon))=[c+d-\varepsilon, c+1-\varepsilon) .
$$

For every $i=2, \ldots, \max (A)$ we have $f^{(i-1)}\left(\Omega_{A}\right) \subset \Omega_{C}$ and therefore

$$
f^{(i)}\left(\Omega_{A}\right)=f^{(i-1)}\left(f\left(\Omega_{A}\right)\right)=[c+d-\varepsilon, c+1-\varepsilon)-(i-1) \varepsilon .
$$

We can thus write

$$
\begin{aligned}
\max (A) & =\max \{k \mid \forall i=1, \ldots, k: c+1-i \varepsilon>c+\varepsilon\} \\
& =\max \{k \mid c+1-k \varepsilon>c+\varepsilon\} \\
& =\left\lfloor\varepsilon^{-1}\right\rfloor-1 .
\end{aligned}
$$

Let us derive the value of $\min (A)$. From the definition of $\min (A)$ and from the properties of the stepping function we have

$$
\begin{aligned}
\min (A) & =\min \left\{k \mid \forall i=1,2, \ldots, k:\left\{\begin{array}{lll}
f^{(i)}\left(\Omega_{A}\right) & \subseteq \Omega_{C} \\
f^{(k+1)}\left(\Omega_{A}\right) & \nsubseteq & \Omega_{C}
\end{array}\right\}\right. \\
& =\min \left\{k \mid \forall i=1, \ldots, k:\left\{\begin{array}{ll}
c+d-i \varepsilon & \geq c+\varepsilon \\
c+d-(k+1) \varepsilon & <c+\varepsilon
\end{array}\right\}\right. \\
& =\max \{k \mid c+d-k \varepsilon \geq c+\varepsilon\} \\
& =\left\lfloor d \varepsilon^{-1}\right\rfloor-1 .
\end{aligned}
$$

Using similar argumentation for the interval $\Omega_{B}$ we derive

$$
\begin{aligned}
& \max (B)=\max \{k \mid c+d-(k-1) \varepsilon>c+\varepsilon\}=\left\lceil d \varepsilon^{-1}\right\rceil-1, \\
& \min (B)=\max \{k \mid c+\varepsilon \leq c+1-k \varepsilon\}=\left\lfloor\varepsilon^{-1}\right\rfloor-1 .
\end{aligned}
$$

Using the stepping function we can determine some relations for the densities of factors in the word $u_{\varepsilon, \eta}(\Omega)$, that will help us in proving the main theorem.

Lemma 7.7. Let $0<\varepsilon<\frac{1}{2}, \eta>0$ be irrational numbers, and let $\Omega$ be an interval of length $1-\varepsilon<|\Omega| \leq 1$. Then

$$
\varrho_{C}>\varrho_{B}, \quad \varrho_{B C}=\varrho_{C B}=\varrho_{B}, \quad \text { and } \quad \varrho_{A B}=\varrho_{B A}=0 .
$$


Proof. The fact $\varrho_{C}>\varrho_{B}$ is obvious since $\varepsilon<\frac{1}{2}$. Let us determine the set $\Omega_{C B}$. We have $x \in \Omega_{C B}$ if $x, \in \Omega_{C}$ and $f(x) \in \Omega_{B}$, hence $\Omega_{C B}=$ $\Omega_{C} \cap f^{-1}\left(\Omega_{B}\right)$. From the properties of the given stepping function it is clear that $f^{-1}\left(\Omega_{B}\right) \subset \Omega_{C}$ and therefore $\Omega_{C B}=f^{-1}\left(\Omega_{B}\right)$. The function $f$ is piecewise linear with slope 1 and thus $\left|\Omega_{C B}\right|=\left|f^{-1}\left(\Omega_{B}\right)\right|=\left|\Omega_{B}\right|$. This implies $\varrho_{C B}=\varrho_{B}$. All the other relations can be derived in a similar way.

Proof of Theorem 7.5. Let $0<\varepsilon, \hat{\varepsilon}<\frac{1}{2}, \eta, \hat{\eta}>0,1-\varepsilon<|\Omega| \leq 1,1-\hat{\varepsilon}<$ $|\hat{\Omega}| \leq 1$. The sequences $u_{\varepsilon, \eta}(\Omega)$ and $u_{\hat{\varepsilon}, \hat{\eta}}(\hat{\Omega})$ are bidirectional infinite words in the alphabet $\{A, B, C\}$. If $u_{\varepsilon, \eta}(\Omega) \stackrel{c}{\sim} u_{\hat{\varepsilon}, \hat{\eta}}(\hat{\Omega})$, then the word $u_{\hat{\varepsilon}, \hat{\eta}}(\hat{\Omega})$ arises from $u_{\varepsilon, \eta}(\Omega)$ by a simple permutation of letters in the alphabet. For the purposes of the proof we shall distinguish the letters $A, B, C$ in the word $u_{\varepsilon, \eta}(\Omega)$ and letters $\hat{A}, \hat{B}, \hat{C}$ in the word $u_{\hat{\varepsilon}, \hat{\eta}}(\hat{\Omega})$. We have 6 permutations on three letters, given by

$$
\begin{aligned}
& h_{1}: \quad A \leftrightarrow \hat{A}, \quad B \leftrightarrow \hat{B}, \quad C \leftrightarrow \hat{C}, \\
& h_{2}: A \leftrightarrow \hat{B}, \quad B \leftrightarrow \hat{A}, \quad C \leftrightarrow \hat{C}, \\
& h_{3}: A \leftrightarrow \hat{C}, \quad B \leftrightarrow \hat{B}, \quad C \leftrightarrow \hat{A}, \\
& h_{4}: A \leftrightarrow \hat{C}, \quad B \leftrightarrow \hat{A}, \quad C \leftrightarrow \hat{B}, \\
& h_{5}: A \leftrightarrow \hat{B}, \quad B \leftrightarrow \hat{C}, \quad C \leftrightarrow \hat{A}, \\
& h_{6}: A \leftrightarrow \hat{A}, \quad B \leftrightarrow \hat{C}, \quad C \leftrightarrow \hat{B} .
\end{aligned}
$$

Obviously, the densities of factors of finite length must correspond in $u_{\varepsilon, \eta}(\Omega)$ and $u_{\hat{\varepsilon}, \hat{\eta}}(\hat{\Omega})$. According to Lemma 7.7, we have $\varrho_{C}>\varrho_{B}$ and $\varrho_{\hat{C}}>\varrho_{\hat{B}}$, thus we may exclude the permutation $h_{6}$.

Let us exclude the permutation $h_{5}$. We use Lemma 7.7 to find that $\varrho_{B}=$ $\varrho_{C B}=\varrho_{\hat{B} \hat{A}}=0$, but this implies that $1-\varepsilon=d$ which is a contradiction. In the same way we exclude $h_{4}$, since $h_{4}$ and $h_{5}$ differ only by interchange of letters with and without hats. For the permutation $h_{3}$ one would have $\varrho_{B}=\varrho_{C B}=\varrho_{\hat{A} \hat{B}}=0$, which is again a contradiction.

The remaining permutations are $h_{1}$ or $h_{2}$. The permutation $h_{1}$ implies $\varrho_{A}=\varrho_{\hat{A}}$ and thus $d=\hat{d}$. Then necessarily also $\varrho_{C}=\varrho_{\hat{C}}$ which implies $\varepsilon=\hat{\varepsilon}$. The combinatorial equivalence of $u_{\varepsilon, \eta}(\Omega)$ and $u_{\hat{\varepsilon}, \hat{\eta}}(\hat{\Omega})$ means that for $\hat{\eta}=\eta$ the sets $\Sigma_{\varepsilon, \eta}(\Omega)$ and $\Sigma_{\hat{\varepsilon}, \hat{\eta}}(\hat{\Omega})$ are geometrically similar. The only way this can happen is that $\hat{\Omega} \cap \mathbb{Z}[\varepsilon]=(x+\Omega) \cap \mathbb{Z}[\varepsilon]$ for some $x \in \mathbb{Z}[\varepsilon]$, cf. Proposition 6.2.

Permutation $h_{2}$ provides

$$
\begin{array}{ccc}
\varrho_{C}=\varrho_{\hat{C}} & \Longrightarrow & \frac{\varepsilon}{d}=\frac{\hat{\varepsilon}}{\hat{d}} \\
\max (A)=\max (\hat{B}) & \Longrightarrow & \left\lfloor\varepsilon^{-1}\right\rfloor=\left[\hat{d} \hat{\varepsilon}^{-1}\right], \\
\min (B)=\min (\hat{A}) & \Longrightarrow & {\left[\varepsilon^{-1}\right\rfloor=\left\lfloor\hat{d} \hat{\varepsilon}^{-1}\right] .}
\end{array}
$$


This is possible only if $\frac{\hat{d}}{\hat{\varepsilon}}=\frac{d}{\varepsilon}=k+1$ for some $k \in \mathbb{N}$. From the relation $\min (A)=\min (\hat{B})$ we then have $\frac{d}{\varepsilon}=\left\lfloor\frac{1}{\hat{\varepsilon}}\right\rfloor$ and therefore $\left\lfloor\frac{1}{\varepsilon}\right\rfloor=\left\lfloor\frac{1}{\hat{\varepsilon}}\right\rfloor=k+1$. The latter gives us finally

$$
\frac{1}{k+2}<\frac{1}{\varepsilon}, \quad \frac{1}{\hat{\varepsilon}}<\frac{1}{k+1}, \quad d=(k+1) \varepsilon, \quad \hat{d}=(k+1) \hat{\varepsilon} .
$$

Therefore both $u_{\varepsilon, \eta}(\Omega)$ and $u_{\hat{\varepsilon}, \hat{\eta}}(\hat{\Omega})$ are $k$-padded Sturmian words derived from Sturmian sequences with slopes $\alpha=\frac{(k+2) \varepsilon-1}{\varepsilon}$ and $1-\alpha=\frac{(k+2) \hat{\varepsilon}-1}{\hat{\varepsilon}}$.

\section{Conclusions}

Infinite ternary words associated with cut-and-project sequences are natural generalizations of Sturmian sequences. Every cut-and-project sequence is characterized by a triple $\varepsilon, \eta, \Omega$, where $\varepsilon, \eta$ are irrational numbers such that $\varepsilon \neq-\eta$, and $\Omega$ is a bounded interval. We have introduced an equivalence on the set of such triples $(\varepsilon, \eta, \Omega)$ using the combinatorial equivalence of corresponding infinite words and we have characterized the equivalence classes, cf. Theorem 7.5.

According to the complexity we may distinguish between quasisturmian sequences with complexity $n+$ const., and words of complexity $2 n+1$. We have shown that a cut-and-project sequence has the complexity $n+$ const., if and only if the length of the acceptance interval $\Omega$ belongs to $\mathbb{Z}[\varepsilon]$. Rauzy graphs $\left(\Gamma_{n}\right)_{n \in \mathbb{N}}$ associated to an infinite word of complexity $2 n+1$ are starting from an $n_{0}$ of the type 2-2, and therefore the cut-and-project sequences are different from those studied in [4]. This after all agrees with the fact that words associated with cut-and-project sequences are codings of three interval exchange transformations, whereas Arnoux-Rauzy sequences are not.

It is interesting to study the invariance of symbolic sequences under morphisms (substitutions). If $\Sigma_{\varepsilon, \eta}(\Omega)$ has a self-similarity factor (there exists $\gamma>1$ such that $\gamma \Sigma_{\varepsilon, \eta}(\Omega) \subset \Sigma_{\varepsilon, \eta}(\Omega)$ ), we can decide about existence of such a morphism. Imposing the requirement of self-similarity implies that $\varepsilon$ is a quadratic integer and $\eta=-\varepsilon^{\prime}$, i.e., the algebraic conjugate of $\varepsilon$. Moreover, $\gamma$ must be a quadratic Pisot number in the same algebraic field, $\gamma \in \mathbb{Q}(\varepsilon)[6]$. In [5] there is a necessary and sufficient condition so that a self-similar cut-and-project sequence is invariant under a morphism. In [1] Adamczewski studies a weaker property, in particular he determines when a coding of a 3-interval exchange is substitutive. Similar question is treated in [16] by different methods. An algorithm for construction of substitution rules for cut-and-project sequences was implemented by Jan Patera, it is available at $[20]$. 


\section{Acknowledgements}

The authors are grateful to the referee for useful suggestions. E.P. and Z.M. are grateful for the hospitality of CRM, Université de Montréal, and L.S.G. is grateful for the hospitality of the Department of Mathematics, FNSPE, Czech Technical University, where parts of the work were done. The authors acknowledge the financial support of the Grant Agency of the Czech Republic, GA 201/01/0130. L.S.G. and Z.M. acknowledge the financial support by NSERC of Canada.

\section{References}

[1] B. AdAMCZEwsKr, Codages de rotations et phénomènes d'autosimilarité. J. Théor. Nombres Bordeaux 14 (2002), 351-386.

[2] P. Alessandri, V. Berthé, Autour du théorème des trois longueurs. Enseign. Math. 44 (1998), 103-132.

[3] J. P. Allouche, Sur la complexité des suites infinies. Bull. Belg. Math. Soc. 1 (1994), 133-143.

[4] P. ARnoux, G. RAuzy, Représentation géométrique de suites de complexité 2n+1. Bull. Soc. Math. France 119 (1991), 199-215.

[5] P. Balážı, Z. Masáková, E. Pelantová, Cut-and-project sequences invariant under morphism. In preparation, Czech Technical University, (2003)

[6] P. Balážı, E. Pelantová, Selfsimilar Cut-and-project Sequences. To be published in Proceedings of Group 24, Paris 2002.

[7] V. BERTHÉ, Sequences of low complexity: automatic and sturmian sequences. Topics in Symbolic Dynamics and Applications, Eds. F. Blanchard, A. Maass, A. Nogueira, Cambridge Univ. Press (2000), 1-34.

[8] J. Cassaigne, Sequences with grouped factors. Developments in Language Theory III, Thessaloniki, Aristotle University of Thessaloniky (1998), 211-222. Available at ftp://iml.univ-mrs.fr/pub/cassaigne/publis/grouped.ps.gz.

[9] E.M. Coven, G.A. HeduUnd, Sequences with minimal block growth. Math. Systems Theory 7 (1973), 138-153.

[10] S. Ferenczi, Complexity of Sequences and Dynamical Systems. Discrete Math. 206 (1999), 663-682.

[11] S. Ferenczi, C. Holton, L.Q. ZAMBoni, Structure of three interval exchange transformations I: An arithmetic study. Ann. Inst. Fourier (Grenoble) 51 (2001), 861-901. http://citeseer.nj.nec.com/article/ferenczi01structure.html

[12] R.L. Graham, D.E. Knuth, O. Patashik, Concrete mathematics. A foundation for computer science. Second edition, Addison Wesley, Reading MA, 1994.

[13] M. Langevin, Stimulateur cardiaque et suites de Farey. Period. Math. Hungar. 23 (1991), 75-86.

[14] M. LothaIRE, Algebraic Combinatorics on Words. Chapter 2: Sturmian words, by J. Berstel, P. Séébold, Cambridge University Press, (2002), 45-110.

[15] W. F. Lunnon, P. A. B. Pleasants, Characterization of two-distance sequences. J. Austral. Math. Soc. (Series A) 53 (1992), 198-218.

[16] Z. Masáková, J. Patera, E. Pelantová, Substitution rules for aperiodic sequences of the cut-and-project type. J. Phys. A: Math. Gen. 33 (2000), 8867-8886.

[17] M. Morse, G. A. Hedlund, Symbolic dynamics II: Sturmian trajectories. Amer. J. Math. 62 (1940), 1-42.

[18] R.V. Moody, Meyer sets and their duals, in Mathematics of Long Range Aperiodic Order. Proc. NATO ASI, Waterloo, 1996, ed. R. V. Moody, Kluwer (1996), 403-441.

[19] R. V. MOODY, J. PATERA, Densities, minimal distances, and coverings of quasicrystals. Comm. Math. Phys. 195 (1998), 613-626.

[20] JAN PATERA, http://kmlinux.fjfi.cvut.cz/־patera/makerule.cgi. 
[21] N. B. Slatter, Gaps and steps for the sequence $n \theta \bmod 1$. Proc. Camb. Phil. Soc. 63 (1967), 1115-1123.

[22] H. WEYL, Über die Gleichungverteilung von Zahlen mod. Eins. Math. Ann. 77 (1916), 313-352.

Louis-Sébastien Guimond

Department of Mathematics and Statistics

McGill University, Burnside Hall

805 Sherbrooke Street West

Montréal (Québec) H3A 2K6, Canada

E-mail : guimondomath.mcgill.ca

\section{Zuzana MASÁKovÁ}

Department of Mathematics

Faculty of Nuclear Sciences and Physical Engineering

Czech Technical University, Trojanova 13

12000 Praha 2, Czech Republic

E-mail : masakovalkm1.fjfi.cvut.cz

Edita Pelantová

Department of Mathematics

Faculty of Nuclear Sciences and Physical Engineering

Czech Technical University, Trojanova 13

12000 Praha 2, Czech Republic

$E$-mail : pelantovaekm1.fjfi.crut.cz 